\title{
Robust Ordinal Regression for Decision under Risk and Uncertainty *
}

\author{
Salvatore Corrente • Salvatore Greco • \\ Benedetto Matarazzo • Roman Słowiński
}

\begin{abstract}
We apply the Robust Ordinal Regression (ROR) approach to decision under risk and uncertainty. ROR is a methodology proposed within Multiple Criteria Decision Aiding (MCDA) with the aim of taking into account the whole set of instances of a given preference model, for example instances of a value function, which are compatible with preference information supplied by the Decision Maker (DM) in terms of some holistic preference comparisons of alternatives. ROR results in two preference relations, necessary and possible; the necessary weak preference relation holds if an alternative is at least as good as another one for all instances compatible with the DM's preference information, while the possible weak preference relation holds if an alternative is at least as good as another one for at least one compatible instance. To apply ROR to decision under risk and uncertainty we have to reformulate such a problem in terms of MCDA. This is obtained by considering as criteria a set of quantiles of the outcome distribution, which are meaningful for the DM. We illustrate our approach in a didactic example based on the celebrated newsvendor problem.
\end{abstract}

* Please cite as: Corrente Salvatore, Greco Salvatore, Matarazzo Benedetto, Słowiński Roman. Robust Ordinal Regression for Decision under Risk and Uncertainty. Journal of Business Economics, 86(1), 55-83

Salvatore Corrente · Salvatore Greco · Benedetto Matarazzo

Department of Economics and Business, University of Catania, Corso Italia, 55, 95129

Catania, Italy

E-mail: salvatore.corrente@unict.it,salgreco@unict.it,matarazz@unict.it

Salvatore Greco

University of Portsmouth, Portsmouth Business School, Centre of Operations Research and Logistics (CORL), Richmond Building, Portland Street, Portsmouth PO1 3DE,

United Kingdom

Roman Słowiński

Institute of Computing Science, Poznań University of Technology, 60-965 Poznań, and

Systems Research Institute, Polish Academy of Sciences, 01-447 Warsaw, Poland,

E-mail: roman.slowinski@cs.put.poznan.pl 
Keywords Multiple criteria decision aiding - Robust ordinal regression • Decision under risk and uncertainty · Additive value functions · Outranking methods

\section{Introduction}

Multiple Criteria Decision Aiding (MCDA) concerns decision problems where an alternative $a$, belonging to a finite set of alternatives $A=\{a, b, c, \ldots\}$, is evaluated by a consistent [58] family of criteria $G=\left\{g_{1}, \ldots, g_{j}, \ldots, g_{m}\right\}$ (see [20] for a collection of state of the art surveys on MCDA). There are several types of MCDA problems, the most important of which are:

- choice problems, where the aim is to select one or more alternatives from $A$ considered the best,

- ranking problems, where the aim is to order, partially or totally, all alternatives from the best to the worst,

- sorting problems, where the aim is to assign all alternatives to one or more contiguous, preferentially ordered categories.

In this paper, we want to use MCDA to deal with decision under risk and uncertainty. Let us remember that a distinction between risk and uncertainty has been advocated by Knight [45] who writes that "The essential fact is that 'risk' means in some cases a quantity susceptible of measurement". Thus, this distinction is based on the possibility to measure the credibility of future events by means of some probability (for a critical discussion on this topic see [47]). In general, in decision under risk and uncertainty, acts from a set $F$ are described in terms of the consequences in a set $X$ corresponding to a set $S$ of exhaustive and mutually exclusive states of the world. Each subset $E \subseteq S$ of states of the world is called event. More precisely, each act $f \in F$, is a function $f: S \rightarrow X$ that assigns to each state of the world $s \in S$ the consequence $f(s) \in X$ obtained if $f$ is selected and $s$ is verified. For the sake of simplicity, let us consider a finite set $S$. In this context, a probability is a function $p: 2^{S} \rightarrow[0,1]$ such that

i) $p(\emptyset)=0$ and $p(S)=1$,

ii) for all $E, E^{\prime} \subseteq S$ such that $E \cap E^{\prime}=\emptyset, p\left(E \cup E^{\prime}\right)=p(E)+p\left(E^{\prime}\right)$.

For each event $E \subseteq S, p(E)$ represents its credibility. One can distinguish between objective probability, exogenously given, and subjective probability, that represents the credibility that a given Decision Maker (DM) assigns to each event $E \subseteq S$ as revealed by her preferences. In this case, for all $E, E^{\prime} \subseteq S$, $p(E)$ is greater than $p\left(E^{\prime}\right)$ if and only if for all $x, y \in X$, whenever the DM prefers $x$ to $y$, then she also prefers the act $x E y$ (giving outcome $x$ if $E$ is verified and $y$ otherwise), to the act $x E^{\prime} y$ (giving outcome $x$ if $E^{\prime}$ is verified and $y$ otherwise). In fact, in the following we shall consider also non additive probabilities, i.e. probabilities for which above property $i i$ ) is replaced by the following weaker monotonicity property: 
iii) for all $E \subseteq E^{\prime} \subseteq S, p(E) \leq p\left(E^{\prime}\right)$.

We shall consider also qualitative probabilities, i.e. probabilities that are expressed on some ordinal scale $L=\left\{l_{0}, l_{1}, \ldots, l_{r}\right\}$ such that for all $i=1, \ldots, r$ $l_{i}$ denotes a greater degree of probability than $l_{i-1}$, with $l_{0}$ representing the credibility that an event will not be verified for sure, and $l_{r}$ the credibility that an event will be certainly verified.

To fix the ideas, let us consider the following example. An economic agent is evaluating a certain number of possible investments $F$, the profits of which depend on the realization of one state of the world in the set $S=\left\{s_{1}, s_{2}, s_{3}\right\}$. For example, for the investment $f \in F$ the profit is $100,000 €$ in $s_{1}, 130,000 €$ in $s_{2}$ and $150,000 €$ in $s_{3}$, that is $f\left(s_{1}\right)=100,000 €, f\left(s_{2}\right)=130,000 €$ and $f\left(s_{3}\right)=150,000 €$. To evaluate comprehensively each investment from $F$ and to compare them, it is not enough to know what is the profit in each state of the world, but other information is necessary. First of all, it is necessary to know which is the probability of each of the three states of the world. In this sense, one possibility is that the economic agent knows a priori the probabilities of each state of the world, for example $p_{1}=20 \%, p_{2}=30 \%$ and $p_{3}=50 \%$ for $s_{1}, s_{2}$ and $s_{3}$, respectively. This is the case of an objective additive probability. It is also possible that, due to some severe uncertainty on the realization of the states of the world, only a non-additive probability is given. For example, due to lack of knowledge about possible realization of $s_{1}$ or $s_{2}$, one can consider a probability of $p_{1}=10 \%$ for $s_{1}, p_{2}=15 \%$ for $s_{2}$, while the probability that one between $s_{1}$ and $s_{2}$ is realized is $p_{12}=50 \%$, which is greater than $p_{1}+p_{2}$. It is worthwhile to remember that nonadditive probability is related to ambiguity for non perfect knowledge of the probability of events suggested by the famous Ellsberg paradox [19], in which in one box there are 90 balls, 30 of which are red and 60 are black or yellow in an unknown proportion. In this case it is quite natural to assign a probability of $1 / 3$ to extract a red ball (because we know that $1 / 3$ of the balls are red), a probability of $2 / 3$ to extract a black or yellow ball (because, even if their proportion is unknown, we know that $2 / 3$ of the balls are black or yellow), a probability let us say of $1 / 5$ to extract a black ball and a probability of $1 / 5$ to extract a yellow ball (because, even if we know that in total black and yellow balls are the $2 / 3$ of the balls in the box, we do not know exactly their proportion). It is also possible that the economic agent does not know a priori any probability, but she has some preferences on acts related to states of the world. For example, she could consider indifferent an act giving $100 €$ on $s_{1}$ and nothing otherwise, and an act giving with certainty $40 €$; therefore, assuming that $40 €$ should be the expected value of the return for the considered act (i.e. $100 € \times p_{1}=40 €$ ), one could induce that the economic agent implicitly assigns a probability of $40 \%$ to $s_{1}$. In this case, we have a subjective probability, which can be both additive or nonadditive. Another possible situation is that the economic agent could not know an a priori "objective" probability and, also, she could not have a subjective probability, because, for example she would not be able or could not want to say if she would exchange an act giving $100 €$ on $s_{1}$ and 
nothing otherwise, with an act giving with certainty $40 €$ or another monetary amount. However, the economic agent could accept to give some qualitative evaluations of the probability of $s_{1}, s_{2}$ and $s_{3}$. For example, she could say that $s_{1}$ and $s_{2}$ have a small probability, $s_{3}$ has a medium probability, the event $\left\{s_{1}, s_{2}\right\}$ has also a medium probability, while the events $\left\{s_{1}, s_{3}\right\}$ and $\left\{s_{2}, s_{3}\right\}$ have a high probability. In this case we have qualitative probability expressed on a scale $L$ having among its elements "small", "medium", "high".

Let us now remember that several models of decision under risk and uncertainty have been proposed in the literature. In this context, the basic model is the expected utility model which assigns to each act $f \in F$ the value

$$
\mathbb{E}_{P}(f)=\sum_{s_{i} \in S} u\left(f\left(s_{i}\right)\right) p_{i}
$$

with $u: X \rightarrow \mathbb{R}$ being a utility function representing tastes of the DM on $X$.

The expected utility model has been considered in case of objective probabilities [64], subjective probability [60] and both subjective and objective probabilities [2]. To take into account nonadditive probability, a generalization of the expected utility model based on the Choquet integral [10] has been considered [61]. Other generalizations representing ambiguity for imperfect knowledge of probabilities take into account a plurality of probabilities $[4,27$, 28].

To clarify the difference of our approach with respect to this literature, let us remember that there are four main approaches to decision making (the first three discussed in [3] and the fourth one proposed in [57]):

- the normative approach, that studies decisions on the basis of general axioms of rationality,

- the descriptive approach, that investigates through experiments how real decisions are taken pointing out some systematic deviations from rationality,

- the prescriptive approach, that aims at avoiding the systematic deviations highlighted by the descriptive approach from the rationality postulated by the first approach $[35,59]$,

- the costructive approach, aiming at supporting a DM to construct her preferences in complex decision problems, especially when a plurality of points of view, technically called criteria, are involved.

While most of the literature on decision under risk and uncertainty has adopted the first three approaches, we would like to propose a methodology related to the fourth approach. In this sense, it is fundamental that the decision model is expressed in terms that can be easily understood by the DM, who has to find in the recommendation of the decision process the arguments useful to explain and justify to herself and to other subjects the suggested decision. In this perspective, the concept of quantile appears to be particuarly useful. Indeed, especially when the states of the world are infinite and the probability distribution is given by means of some analytical formulation, not easily comprehensible even by a DM with a technical background, it is much 
more reasonable to take into account some meaningful probability thresholds and to reformulate the probability in terms of quantiles. In fact, in our real life experience, very often in front of some risks we reason answering to questions such as: "Which is the gain I can get with probability of $90 \%$ (or $75 \%$, or $50 \%$, or any other relevant probability threshold)?" or "Which is the loss I can incur with probability of $10 \%$ (or $25 \%$ or $50 \%$, or any other relevant probability threshold)?" Quantiles have been extensively used in finance where, for example, the $p \%$ Value at Risk is the worst $p \%$ loss [38]. Some axiomatic foundations of decision based on quantiles have also been proposed in economics $[9,48,56]$. What is different in our proposal is that, following [31] (see also [50]), we suggest to consider a certain number of quantiles rather than only one. In this sense, a set of quantiles can be interpreted as a parsimonious but effective representation of the whole information contained in a probability distribution, taking into account the limited capability of human mind. Therefore, in line with the famous Miller's article "The Magical Number Seven, Plus or Minus Two: Some Limits on Our Capacity for Processing Information" [51], we suggest a number of quantiles between 5 and 9 because, as argued in that article, this is the number of objects that a human brain can handle due to the limits of one-dimensional absolute judgment and to the limits of short-term memory. More formally, we propose to deal with decision problems in case of risk and uncertainty considering a set of quantiles on the domain of criterion $g_{j} \in G$ corresponding to a set of meaningful probability levels $P_{j}=\left\{\pi_{1}, \ldots, \pi_{h_{j}}\right\}$. For each $\pi \in P_{j}$, a value function $g_{j}^{\pi}: A \rightarrow \mathbb{R}$ is defined such that, for each $a \in A$, $g_{j}^{\pi}(a)=x$ means that, fixed the probability $\pi$, the minimum value got by $a$ on criterion $g_{j}$ with probability at least $\pi$ is $x$. Therefore, for all $j=1, \ldots, m$, for all $\pi \in P_{j}$ and for all $a \in A$,

$$
g_{j}^{\pi}(a)=x \Leftrightarrow P\left(g_{j}(a) \geq x\right) \geq \pi,
$$

where $P$ is a probability distribution on the space of values attainable by each alternative from $A$ on criteria from $G$. Therefore, we propose to deal with uncertainty in MCDA by replacing uncertain criteria $g_{j}$ by the corresponding set of value functions $g_{j}^{\pi}, \pi \in P_{j}$.

Let us note that, while "alternatives" are the objects of the decision in MCDA, they are called "acts" in the framework of decision under risk and uncertainty. Since our aim is dealing with the decision under risk and uncertainty using the MCDA framework, in the following, we shall use the term "alternative".

Continuing the previous example and, considering the following quantiles $P_{j}=\left\{\pi_{1}, \pi_{2}, \pi_{3}\right\}=\{30 \%, 60 \%, 90 \%\}$ relative to the criterion profit denoted by $f\left(s_{1}\right)=100,000 €, f\left(s_{2}\right)=130,000 €$ and $f\left(s_{3}\right)=150,000 €$, we have that $g_{j}^{30 \%}(a)=150,000 €, g_{j}^{60 \%}(a)=130,000 €$, and $g_{j}^{90 \%}(a)=100,000 €$. Let us observe also that, as explained in [30], quantiles can be used also in case of nonadditive probability and even qualitative probability.

As shown in [30], this approach can be extended to deal also with time preferences. In this case, one considers a set of meaningful time epochs $\mathbb{T}=$ $\left\{t_{1}, \ldots, t_{r}\right\}$, such that for each criterion $g_{j} \in G, \pi \in P_{j}$ and $t \in \mathbb{T}$, a function 
$g_{j}^{\pi, t}$ is defined. For each $a \in A, g_{j}^{\pi, t}(a)=x$ means that, with respect to criterion $g_{j}$, action $a$ gets at least value $x$ within time epoch $t$ with a probability at least $\pi$.

For the sake of simplicity, in this paper we consider only the case of a single criterion without considering time preferences, deferring to future research discussion of these points. As already observed in [30], this new formulation of decision under risk and uncertainty as a multiple criteria decision problem, can be dealt with other methods proposed in MCDA, such as value function methods [43], outranking-based methods [8,25], decision rule methods inferred by Dominance-based Rough Set Approach [32,62,63], interactive multiobjective optimization and evolutionary multiobjective optimization methods [6]. In this paper we shall describe how to handle the new formulation of the multiple criteria decision problem by using an MCDA methodology that is particularly in line with the principle of the constructive approach. This is the Robust Ordinal Regression (ROR) $[12,13,33]$. ROR, as classical ordinal regression [36], asks the DM to supply some preference information, for example in terms of some pairwise comparisons between alternatives on which the preference of the DM is certain. Both, ordinal regression and ROR aim at supporting the DM giving some recommendations on the basis of the provided preference information. However, adopted a given class of preference models, while ordinal regression selects only one instance of the preference model among all representing the preference information provided by the DM, ROR takes into account the whole set of instances of the considered preference model compatible with the preference information. For example, if the adopted model is the additive multiple attribute utility function, while ordinal regression selects a single utility function among all representing the preference information, ROR considers the whole set of utility functions compatible with the preference information. To give account of this plurality of instances, ROR presents recommendations in terms of necessary and possible preference relations. Given two alternatives $a$ and $b, a$ is (weakly) necessarily preferred to $b$, if $a$ is at least as good as $b$ for all instances of the preference model compatible with the preference information supplied by the DM, while $a$ is (weakly) possibly preferred to $b$ if $a$ is at least as good as $b$ for at least one compatible instance of the preference model. A great advantage of ROR is that, considering the whole set of compatible instances, it avoids to arrive to premature conclusions on preferences as it is possible with classical ordinal regression. Indeed, different compatible instances of the preference model can give different preference relations, and therefore, it is always arbitrary to some extent to select only one of them. Instead, it is more cautious to separate stable preferences, those resulting from all compatible instances, from unstable preferences, that hold for some compatible instance but do not hold for some other ones. Necessary and possible preferences so obtained can be presented to the DM who can react by accepting them or criticizing them. If the DM accepts the necessary and the possible preferences, and if she is satisfied by the results, she can conclude the decision process; on the contrary, she can add new preference information in order to obtain more precise results, i.e., a richer necessary preference relation. Actually, if the DM 
criticizes the necessary and the possible preferences, she can modify some preference information in order to get results better representing her preferences. In any case, ROR permits to open a discussion with the DM giving her the possibility to reflect on the decision problem, and to arrive to a mature and convincing decision. For all these good properties, ROR has been applied to several MCDA preference models, namely additive utility functions [14,21,33], ELECTRE methods [15,29], PROMETHEE methods [39], Choquet integral [1] and an enriched form of the additive utility function to take into account interaction between criteria [34]. Thus, the constructive MCDA methodology that we present in this approach is based on two pillars that give it its essential properties: on one hand, the representation of the probability in terms of quantiles ensures the easy understanding by the DM while, on the other hand, ROR permits a prudent inference of further preferences on the basis of the current preference information supplied by the DM.

The paper is structured as follows: in Section 2 we introduce the notation used in the paper; Section 3 recalls the different preference models used in MCDA and the Robust Ordinal Regression (ROR); GRIP and ELECTRE ${ }^{G K M S}$ are briefly described in Section 4; in Section 5, the new procedure is applied to the newsvendor problem, while conclusions and further directions of research are given in Section 6 .

\section{Notation}

In this section we introduce the notation used in the paper. More details on the meaning of the parameters will be provided in the sections describing the models in which these parameters are involved.

- $A=\{a, b, c, \ldots\}$ - a finite set of $n$ alternatives described over a family $G$ of $m$ evaluation criteria,

- $g_{1}, \ldots, g_{j}, \ldots, g_{m}-m$ evaluation criteria, $g_{j}: A \rightarrow \mathbb{R}$ for all $j \in\{1,2, \ldots, m\}$; the family of criteria $G$ is supposed to be consistent [58], that is exhaustive (all relevant criteria are taken into account), coherent (if two alternatives $a$ and $b$ have the same evaluations on all but one criterion, and $a$ gets an evaluation better than $b$ on the remaining criterion, then $a$ should be preferred to $b$ ), non-redundant (the removal of one criterion from the family makes the new set of criteria not exhaustive);

- $w_{1}, \ldots, w_{j}, \ldots, w_{m}$ - importance coefficients (ELECTRE ${ }^{G K M S}$ only), where $w_{j}$ represents the importance of criterion $g_{j}$ inside the family of criteria $G$;

- $q_{j}, p_{j}$ and $v_{j}, j=1, \ldots, m$, being the indifference, preference and veto thresholds, respectively (ELECTRE ${ }^{G K M S}$ only). 


\section{Preference models and Robust Ordinal Regression}

Since the only information stemming from the evaluations of the alternatives with respect to the different criteria is the dominance relation ${ }^{1}$, three main approaches are used in MCDA to aggregate these evaluations, that are, Multi-Attribute Value Theory (MAVT) [43], outranking methods [8,24] and Dominance-Based Rough Set Approach (DRSA) [32,62]:

- MAVT represents preferences of a DM on a set of alternatives $A$ by an overall value function

$$
U(a)=U\left(g_{1}(a), \ldots, g_{m}(a)\right): \mathbb{R}^{m} \rightarrow \mathbb{R}
$$

such that $a$ is at least as good as $b$ iff $U(a) \geq U(b)$; in the following, we shall suppose that the evaluation criteria are mutually preferentially independent [43] and, consequently, the value function in eq.(1) can be written in an additive way, that is,

$$
U(a)=\sum_{j=1}^{m} u_{j}\left(g_{j}(a)\right)
$$

where $u_{j}: \mathbb{R} \rightarrow \mathbb{R}$ are marginal value functions, for each $j=1, \ldots, m$;

- outranking methods represent preferences of a DM on a set of alternatives $A$ by an outranking relation $S \subseteq A \times A$, such that, $a S b$ iff $a$ is at least as good as $b$;

- DRSA is based on "if,..., then..." decision rules expressed in a natural language for the DM, linking the performances of the alternatives on the considered criteria with a comprehensive judgment of the alternative at hand. For example, "if the consumption of a car is at least $15 \mathrm{~km} / \mathrm{l}$ and its price is at most $10,000 €$, then the car is considered to be good."

In this paper, we shall deal with the first two aggregation approaches.

In order to apply both families of methods, the DM should provide the parameters on which they are based, that are, marginal value functions $u_{j}(\cdot)$, $j=1, \ldots, m$, in MAVT, and weights, indifference, preference and veto thresholds, as well as the cutting level, for the outranking methods. These parameters can be provided by the DM in a direct or in an indirect way $[36,37]$. In the direct one, the DM has to give directly all the values of the parameters involved in the model, while, in the indirect one, the DM gives some preferences on reference alternatives (s)he knows well, from which, parameters compatible with these preferences can be inferred. If the DM provides an indirect preference information, in general, more than one instance of the model (a value function in the MAVT case and a set of weights, thresholds and cutting level in the second one) could be compatible with the preferences she provided. Each of these models provides the same recommendations on the

\footnotetext{
1 Supposing that all criteria are gain criteria, $a$ dominates $b$ if $g_{j}(a) \geq g_{j}(b)$ for all $j=1, \ldots, m$, and there exists at least one $j$ such that $g_{j}(a)>g_{j}(b)$.
} 
reference alternatives but each of them could provide different recommendations on the other alternatives from the whole set $A$. To consider all models compatible with the preferences of the DM, ROR has been proposed $[12,13$, 33 . ROR provides robust recommendations with respect to the problem at hand, building a necessary and a possible preference relation. The necessary and possible preference relations hold between two alternatives $a$ and $b$ if $a$ is at least as good as $b$ for all or, respectively, for at least one instance of the model compatible with the preferences provided by the DM.

\section{GRIP and ELECTRE ${ }^{G K M S}$}

The first method applying ROR concepts is $\mathrm{UTA}^{G M S}$. It is based on the additive value function shown in eq. (2) and it is used to deal with ranking and choice problems. In this section, we shall briefly recall two methods, that are GRIP [23] and ELECTRE ${ }^{G K M S}$ [29]. GRIP is the generalization of the $\mathrm{UTA}^{G M S}$ method taking into account not only pairwise comparisons between alternatives but also information on intensity of preferences between two pairs of alternatives. ELECTRE ${ }^{G K M S}$ is the extension of the ELECTRE IS method under the ROR framework.

\subsection{GRIP}

In the GRIP method, the DM is expected to provide the following indirect preference information both at comprehensive and at partial level with respect to a subset of reference alternatives $A^{R} \subseteq A$ :

- A partial preorder $\succsim$ on $A^{R}$ whose meaning is: for $a^{*}, b^{*} \in A^{R}$

$$
a^{*} \succsim b^{*} \Leftrightarrow " a^{*} \text { is at least as good as } b^{*} " \text {. }
$$

- A partial preorder $\succsim^{*}$ on $A^{R} \times A^{R}$, whose meaning is: for $a^{*}, b^{*}, c^{*}, d^{*} \in A^{R}$, $\left(a^{*}, b^{*}\right) \succsim^{*}\left(c^{*}, d^{*}\right) \Leftrightarrow$ " $a^{*}$ is preferred to $b^{*}$ at least as much as $c^{*}$ is preferred to $d^{*}$ ".

- A partial preorder $\succsim_{(i, j)}$ on $A^{R}$ whose meaning is: for $a^{*}, b^{*} \in A^{R}$, $a^{*} \succsim_{(i, j)} b^{*} \Leftrightarrow$ "the marginal value of $a^{*}$ on criterion $g_{i}$ is at least as much as the marginal value of $b^{*}$ on criterion $g_{j}$ ".

- A partial preorder $\succsim_{(i, j)}^{*}$ on $A^{R} \times A^{R}$ whose meaning is: for $a^{*}, b^{*}, c^{*}, d^{*} \in$ $A^{R}$,

$$
\begin{aligned}
\left(a^{*}, b^{*}\right) \succsim_{(i, j)}^{*}\left(c^{*}, d^{*}\right) \Leftrightarrow & \text { " } a^{*} \text { is preferred to } b^{*} \text { on criterion } g_{i} \text { at least as much as } \\
& c^{*} \text { is preferred to } d^{*} \text { on criterion } g_{j}, i, j \in G^{\prime} .
\end{aligned}
$$


Formally, a compatible value function is an additive value function, as that one in eq. (2), satisfying the following set of constraints:

$$
\begin{aligned}
& U\left(a^{*}\right) \geq U\left(b^{*}\right)+\varepsilon \text { if } a^{*} \succ b^{*} \\
& U\left(a^{*}\right)=U\left(b^{*}\right) \quad \text { if } \quad a^{*} \sim b^{*} \\
& \left.\begin{array}{l}
U\left(a^{*}\right)-U\left(b^{*}\right) \geq U\left(c^{*}\right)-U\left(d^{*}\right)+\varepsilon \\
U\left(c^{*}\right) \geq U\left(d^{*}\right)+\varepsilon
\end{array}\right\} \quad \text { if } \quad\left(a^{*}, b^{*}\right) \succ^{*}\left(c^{*}, d^{*}\right) \\
& U\left(a^{*}\right)-U\left(b^{*}\right)=U\left(c^{*}\right)-U\left(d^{*}\right) \text { if }\left(a^{*}, b^{*}\right) \sim^{*}\left(c^{*}, d^{*}\right) \\
& u_{i}\left(a^{*}\right) \geq u_{j}\left(b^{*}\right)+\varepsilon \text { if } a^{*} \succ_{(i, j)} b^{*} \\
& u_{i}\left(a^{*}\right)=u_{j}\left(b^{*}\right) \text { if } a^{*} \sim_{(i, j)} b^{*} \\
& u_{i}\left(a^{*}\right)-u_{i}\left(b^{*}\right) \geq u_{j}\left(c^{*}\right)-u_{j}\left(d^{*}\right)+\varepsilon \text { if }\left(a^{*}, b^{*}\right) \succ_{(i, j)}^{*}\left(c^{*}, d^{*}\right) \\
& u_{i}\left(a^{*}\right)-u_{i}\left(b^{*}\right)=u_{j}\left(c^{*}\right)-u_{j}\left(d^{*}\right) \text { if }\left(a^{*}, b^{*}\right) \sim_{(i, j)}^{*}\left(c^{*}, d^{*}\right) \\
& u_{j}\left(x_{j}^{k}\right) \geq u_{j}\left(x_{j}^{k-1}\right), j=1, \ldots, m, k=1, \ldots, m_{j} \text {, } \\
& u_{j}\left(x_{j}^{0}\right)=0, j=1, \ldots, m, \text { and } \sum_{j=1}^{m} u_{j}\left(x_{j}^{m_{j}}\right)=1
\end{aligned}
$$

where

- $\succ, \succ^{*}, \succ_{(i, j)}$ and $\succ_{(i, j)}^{*}$ are the asymmetric parts of $\succsim \succsim^{*}, \succsim_{(i, j)}$ and $\succsim_{(i, j)}^{*}$, while $\sim, \sim^{*}, \sim_{(i, j)}$ and $\sim_{(i, j)}^{*}$ are their symmetric parts; for example, $a \succ b$ iff $a \succsim b$ and $n o t(b \succsim a)$, while $a \sim b$ iff $a \succsim b$ and $b \succsim a$;

- $x_{j}^{k}, \underset{k=0}{k}, \ldots, m_{j}$, are all different evaluations of the alternatives from $A$ on criterion $g_{j}, j=1 \ldots, m$; the values $x_{j}^{k}, k=0, \ldots, m_{j}$, are increasingly ordered, i.e., $x_{j}^{0}<x_{j}^{1}<\ldots<x_{j}^{m_{j}}$; in particular, $x_{j}^{0}=\min _{a \in A} g_{j}(a)$, while $x_{j}^{m_{j}}=\max _{a \in A} g_{j}(a)$;

- $\varepsilon$ is an auxiliary variable used to convert the strict inequality constraints, translating the preferences of the DM, in weak inequality constraints. For example, the global preference of $a^{*}$ over $b^{*}$, translated to the inequality $U(a)>U(b)$, is converted to the constraint $U(a) \geq U(b)+\varepsilon$.

To check if there exists at least one value function compatible with the preferences provided by the DM, one has to solve the following LP problem:

$$
\varepsilon\left(A^{R}\right)=\max \varepsilon, \text { subject to } E^{A^{R}} \text {. }
$$

If $E^{A^{R}}$ is feasible and $\varepsilon\left(A^{R}\right)>0$, then there exists at least one instance of the model compatible with the preferences provided by the DM. Otherwise, pieces of preference information causing the infeasibility need to be checked by means of some of the methods presented in [53].

Given $a, b \in A$, and the following sets of constraints

$$
\left.\left.\begin{array}{l}
U(b) \geq U(a)+\varepsilon, \\
E^{A^{R}}
\end{array}\right\} E^{N}(a, b), \quad \begin{array}{l}
U(a) \geq U(b), \\
E^{A^{R}}
\end{array}\right\} E^{P}(a, b)
$$

we can say the following: 
- $a$ is necessarily preferred to $b\left(a \succsim^{N} b\right)$, iff $E^{N}(a, b)$ is infeasible or, if $E^{N}(a, b)$ is feasible and $\varepsilon^{N}(a, b) \leq \widetilde{0}$, where $\varepsilon^{N}(a, b)=\max \varepsilon$, subject to $E^{N}(a, b)$;

- $a$ is possibly preferred to $b\left(a \succsim^{P} b\right)$, iff $E^{P}(a, b)$ is feasible and $\varepsilon^{P}(a, b)>0$, where $\varepsilon^{P}(a, b)=\max \varepsilon$, subject to $E^{P}(a, b)$.

More details from the computational point of view on the necessary and possible preference relations could be found in [11], while for some properties of the necessary and possible preference relations, as well as for an axiomatic basis of the same preference relations, the interested reader is referred to [33] and [26], respectively.

In general, in ranking and choice problems, one needs to assign a real number to each alternative being representative of its value in the problem at hand. For this reason, to summarize the results obtained by ROR, a representative value function can be computed $[22,40]$. It is obtained in two steps maximizing, at first, the difference $U(a)-U(b)$ for the pairs of alternatives $(a, b)$ such that $a$ is strictly necessarily preferred to $b$ and, then, minimizing the difference $U(a)-U(b)$ for the pairs of alternatives $(a, b)$ such that $a$ is possibly preferred to $b$ and $b$ is possibly preferred to $a$. From a computational point of view, the two steps are the following:

step 1) solve the LP problem:

$$
\left.\begin{array}{l}
\max \varepsilon, \text { subject to } \\
U(a) \geq U(b)+\varepsilon \text { if } a \succsim^{N} b \text { and } \operatorname{not}\left(b \succsim^{N} a\right), \\
E^{A^{R}} ;
\end{array}\right\}
$$

step 2) denoting by $\varepsilon_{1}$ the value of $\varepsilon$ optimizing the LP problem solved in step 1), solve the following LP problem:

$$
\begin{aligned}
& \min \delta \text {, subject to }
\end{aligned}
$$

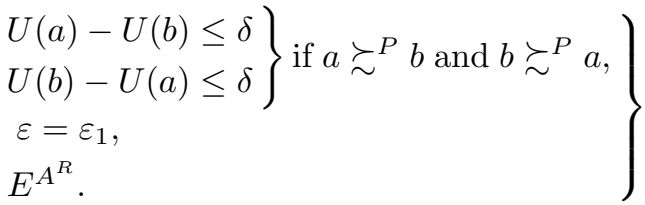

\subsection{ELECTRE EKMS $^{\mathrm{GKM}}$}

Before introducing the ELECTRE ${ }^{G K M S}$ method, we recall the basic aspects of the ELECTRE IS method.

Given the weights of criteria $w_{1}, \ldots, w_{m}$, such that $w_{j} \geq 0$ for all $j=1, \ldots, m$, and $\sum_{j=1}^{m} w_{j}=1$, and the indifference and preference thresholds, $q_{j}(a)$ and $p_{j}(a)$, for all gain-type criteria $g_{j}$, and for all $a \in A$, ELECTRE IS builds, for 
each criterion $g_{j}$, and for each pair of alternatives $(a, b)$, the partial concordance index

$$
\varphi_{j}(a, b)= \begin{cases}1 & \text { if } g_{j}(b)-g_{j}(a) \leq q_{j}(a) \\ \frac{g_{j}(a)-\left[g_{j}(b)-p_{j}(a)\right]}{p_{j}(a)-q_{j}(a)} & \text { if } q_{j}(a) \leq g_{j}(b)-g_{j}(a) \leq p_{j}(a) \\ 0 & \text { if } g_{j}(b)-g_{j}(a) \geq p_{j}(a) .\end{cases}
$$

The indifference and preference thresholds $q_{j}(a)$ and $p_{j}(a)$ have the following meaning:

- $q_{j}(a)$ is the greatest difference between $g_{j}(b)$ and $g_{j}(a)$ compatible with their indifference on criterion $g_{j}$,

- $p_{j}(a)$ is the smallest difference between $g_{j}(b)$ and $g_{j}(a)$ compatible with the preference of $b$ over $a$ on criterion $g_{j}$.

$\varphi_{j}(a, b) \in[0,1]$ for all $j=1, \ldots, m$, and for each $(a, b) \in A \times A$. It represents the degree of preference of $a$ over $b$ with respect to criterion $g_{j}$ and it is a nonincreasing function of the difference $g_{j}(b)-g_{j}(a)$. Observe that, for the sake of simplicity, the indifference and preference thresholds could be considered constant for each alternative $a$ and, therefore, independent of the evaluations $g_{j}(a)$.

After building the partial concordance indices $\phi_{j}(a, b)$, ELECTRE IS builds the comprehensive concordance index $C(a, b)$ for each $(a, b) \in A \times A$, defined as follows:

$$
C(a, b)=\sum_{j=1}^{m} \varphi_{j}(a, b) \times w_{j} .
$$

$C(a, b) \in[0,1]$ and it represents the degree of preference of $a$ over $b$. The outranking relation of the ELECTRE IS method is therefore based on the concordance and the non-discordance tests; the concordance test is verified if $C(a, b) \geq \lambda$, where $\lambda \in[0.5,1]$ is called the cutting level and it represents the minimum coalition of criteria necessary to the outranking of $a$ over $b$. The non-discordance test is verified if, for all $j=1, \ldots, m, g_{j}(b)-g_{j}(a)<v_{j}(a)$ that is, for each criterion $g_{j}$ the difference in the performances of $b$ and $a$ on criterion $g_{j}$ is lower than the veto threshold $v_{j}(a)$. From a formal point of view, we can therefore write that

$a S b$ iff $C(a, b) \geq \lambda$ and $g_{j}(b)-g_{j}(a)<v_{j}(a)$, for all $j=1, \ldots, m$.

The negation of the preference relation $S$ will be denoted by $S^{C}$ and, of course, it will be true that $a S^{C} b$ if at least one of the concordance and the nondiscordance test is not fulfilled.

As already observed in Section 3, the application of the ELECTRE methods involves the knowledge of several parameters that are, weights of criteria, indifference, preference and veto thresholds and the cutting level. In order to induce a set of parameters, the DM can provide the following direct or indirect preference information regarding the evaluation criteria and the comparison between alternatives: 
1) the weight $w_{j}$ of criterion $g_{j}$ belongs to the interval $\left[w_{j}^{L}, w_{j}^{R}\right]$, where $w_{j}^{L} \leq$ $w_{j}^{R}$,

2) criterion $g_{i}$ is more important than criterion $g_{j}$, that is $w_{i}>w_{j}$,

3 ) the indifference threshold for criterion $g_{j}$ related to alternative $a, q_{j}(a)$, belongs to the interval $\left[q_{j}^{L}(a), q_{j}^{R}(a)\right]$, where $q_{j}^{L}(a) \leq q_{j}^{R}(a)$,

4) the preference threshold for criterion $g_{j}$ related to alternative $a, p_{j}(a)$, belongs to the interval $\left[p_{j}^{L}(a), p_{j}^{R}(a)\right]$, where $p_{j}^{L}(a) \leq p_{j}^{R}(a)$,

5) $a \sim_{j} b$, that is "the difference between $g_{j}(a)$ and $g_{j}(b)$ is non-significant for the DM",

6) $a \succ_{j} b$, that is "the difference between $g_{j}(a)$ and $g_{j}(b)$ is significant for the DM",

7) $a$ outranks $b$, that is $a S b$,

8) $a$ does not outrank $b$, that is $a S^{C} b$.

Details on the constraints translating the preference information provided by the DM can be found in [29] and, for the sake of completeness, also in the Appendix A.

If the preferences of the DM are not inconsistent, in general, more than one instance of the model (weights, thresholds and cutting level) compatible with these preferences could be inferred. Even if there exists different methods aiming to find only one model compatible with the preferences provided by the DM (see for example $[17,52]$ ), in this section we shall concentrate our attention on the ELECTRE ${ }^{G K M S}$ method. ELECTRE ${ }^{G K M S}$ takes into account simultaneously all compatible instances of the outranking model defining, as all ROR methods, a necessary and a possible preference relation as follows:

- $a$ is necessarily preferred to $b$ (denoted by $a S^{N} b$ ), iff $a S b$ for all compatible instances of the model,

- $a$ is possibly preferred to $b$ (denoted by $a S^{P} b$ ), iff $a S b$ for at least one compatible instance of the model.

Analogously, other two preference relations related to the non-outranking can be defined:

- $a$ does not necessarily outrank $b$ (denoted by $a S^{C N} b$ ), iff $a S^{C} b$ for all compatible instances of the model,

- $a$ does not possibly outrank $b$ (denoted by $a S^{C P} b$ ), iff $a S^{C} b$ for at least one compatible instance of the model.

It is obvious that $S^{N} \subseteq S^{P}$ and $S^{C N} \subseteq S^{C P}$. Moreover, it can easily be proved that $a S^{N} b$ iff $\operatorname{not}\left(a S^{C P} b\right)$ and $a \bar{S}^{P} b$ iff $\operatorname{not}\left(a S^{C N} b\right)$. The interested reader could find these and other properties of the four preference relations in [29]. Details on the computations of the necessary $\left(S^{N}\right)$ and possible $\left(S^{P}\right)$ preference relations can be found in $[29]$ and, for the completeness of description, in the Appendix B. 


\section{A didactic example concerning the newsvendor problem}

The newsvendor problem is a classic problem of operational research that was faced for the first time by the economist F.Y.Edgeworth in his paper "The mathematical theory of banking" in 1888 [18]. The newsvendor problem consists in finding the correct amount of a certain resource that should be bought, knowing the probability distribution of demand, in order to maximize the payoffs, from one side, or to minimize the costs from the other side. In the last decade, tens of papers were published about this problem and its application in different situations: buying seasonal goods, making the last buying or last production run decision, setting safety stock levels, setting target inventory levels, selecting the right capacity for a facility or machine, overbooking customers, etc. Some literature reviews of the newsvendor problem are the following [44, $54,55]$.

Now, after formulating the problem in the nice case of the newsboy, we will approach it in a new way, as announced in the introduction.

Let's suppose the newsboy has to choose the number of newspapers to be ordered. If he didn't order enough newspapers he will have a stockout problem, so that some customers will be disappointed and sales and profit will be lost. If he orders too many newspapers, he will loose a certain amount of money. The newsboy's goal is, therefore, to choose the amount of newspapers maximizing his gain. The newsboy evaluates that each newspaper involves the following costs and gains:

- Unit cost, $c=1.4 €$,

- Unit selling price, $p=2 €$,

- Salvage value (estimated worth of a newspaper not sold), $s=1 €$,

- Stockout cost (cost of buying one unit less than the demand), $s_{c}=0.15 €$.

Let's suppose that the newsboy has to choose to order among $5, \ldots, 17$ newspapers, and that the demand can assume values $5,6,7,8,9,10$ with probability $5 \%$, values $11,12,13,14,15,16,17$, with probability $10 \%$, and probability $0 \%$ otherwise. Formally,

$$
p_{r}=\left\{\begin{array}{l}
5 \% \text { if } r \in\{5, \ldots, 10\} \\
10 \% \text { if } r \in\{11, \ldots, 17\} \\
0 \% \text { otherwise }
\end{array}\right.
$$

Denoting by $D$ the demand of newspapers and by $Q$ the number of ordered newspapers, the newsboy's gain, denoted by $G(Q, D)$, is the following:

$$
G(Q, D)=p \cdot \min (Q, D)-c \cdot Q+s \cdot \max (Q-D, 0)-s_{c} \cdot \max (0, D-Q)
$$

Table 1 contains the gain for the newsboy as a function of the demand and of the number of newspapers bought. For each $k=5, \ldots, 17$ and for each 
Table 1: Possible gains of the newsboy depending on the demand and on the number of ordered newspapers expressed in $€$

\begin{tabular}{|c|c|c|c|c|c|c|c|c|c|c|c|c|c|}
\hline$G\left(Q_{k}, D_{j}\right)$ & $D_{5}$ & $D_{6}$ & $D_{7}$ & $D_{8}$ & $D_{9}$ & $D_{10}$ & $D_{11}$ & $D_{12}$ & $D_{13}$ & $D_{14}$ & $D_{15}$ & $D_{16}$ & $D_{17}$ \\
\hline$Q_{5}$ & 3.00 & 2.85 & 2.70 & 2.55 & 2.40 & 2.25 & 2.10 & 1.95 & 1.80 & 1.65 & 1.50 & 1.35 & 1.20 \\
$Q_{6}$ & 2.60 & 3.60 & 3.45 & 3.30 & 3.15 & 3.00 & 2.85 & 2.70 & 2.55 & 2.40 & 2.25 & 2.10 & 1.95 \\
$Q_{7}$ & 2.20 & 3.20 & 4.20 & 4.05 & 3.90 & 3.75 & 3.60 & 3.45 & 3.30 & 3.15 & 3.00 & 2.85 & 2.70 \\
$Q_{8}$ & 1.80 & 2.80 & 3.80 & 4.80 & 4.65 & 4.50 & 4.35 & 4.20 & 4.05 & 3.90 & 3.75 & 3.60 & 3.45 \\
$Q_{9}$ & 1.40 & 2.40 & 3.40 & 4.40 & 5.40 & 5.25 & 5.10 & 4.95 & 4.80 & 4.65 & 4.50 & 4.35 & 4.20 \\
$Q_{10}$ & 1.00 & 2.00 & 3.00 & 4.00 & 5.00 & 6.00 & 5.85 & 5.70 & 5.55 & 5.40 & 5.25 & 5.10 & 4.95 \\
$Q_{11}$ & 0.60 & 1.60 & 2.60 & 3.60 & 4.60 & 5.60 & 6.60 & 6.45 & 6.30 & 6.15 & 6.00 & 5.85 & 5.70 \\
$Q_{12}$ & 0.20 & 1.20 & 2.20 & 3.20 & 4.20 & 5.20 & 6.20 & 7.20 & 7.05 & 6.90 & 6.75 & 6.60 & 6.45 \\
$Q_{13}$ & -0.20 & 0.80 & 1.80 & 2.80 & 3.80 & 4.80 & 5.80 & 6.80 & 7.80 & 7.65 & 7.50 & 7.35 & 7.20 \\
$Q_{14}$ & -0.60 & 0.40 & 1.40 & 2.40 & 3.40 & 4.40 & 5.40 & 6.40 & 7.40 & 8.40 & 8.25 & 8.10 & 7.95 \\
$Q_{15}$ & -1.00 & 0.00 & 1.00 & 2.00 & 3.00 & 4.00 & 5.00 & 6.00 & 7.00 & 8.00 & 9.00 & 8.85 & 8.70 \\
$Q_{16}$ & -1.40 & -0.40 & 0.60 & 1.60 & 2.60 & 3.60 & 4.60 & 5.60 & 6.60 & 7.60 & 8.60 & 9.60 & 9.45 \\
$Q_{17}$ & -1.80 & -0.80 & 0.20 & 1.20 & 2.20 & 3.20 & 4.20 & 5.20 & 6.20 & 7.20 & 8.20 & 9.20 & 10.20 \\
\hline
\end{tabular}

Table 2: Values of $z\left(Q_{k}, D_{j}\right)$ with $k, j=5, \ldots, 17$ expressed in percentage. $z\left(Q_{k}, D_{j}\right)$ represents the probability that buying $Q_{k}$ newspapers the newsboy will gain not less than $G\left(Q_{k}, D_{j}\right)$

\begin{tabular}{|c|c|c|c|c|c|c|c|c|c|c|c|c|c|}
\hline$z\left(Q_{k}, D_{j}\right)$ & $D_{5}$ & $D_{6}$ & $D_{7}$ & $D_{8}$ & $D_{9}$ & $D_{10}$ & $D_{11}$ & $D_{12}$ & $D_{13}$ & $D_{14}$ & $D_{15}$ & $D_{16}$ & $D_{17}$ \\
\hline$Q_{5}$ & 5 & 10 & 15 & 20 & 25 & 30 & 40 & 50 & 60 & 70 & 80 & 90 & 100 \\
$Q_{6}$ & 50 & 5 & 10 & 15 & 20 & 25 & 35 & 45 & 60 & 70 & 80 & 90 & 100 \\
$Q_{7}$ & 100 & 55 & 5 & 10 & 15 & 20 & 30 & 40 & 50 & 65 & 75 & 85 & 95 \\
$Q_{8}$ & 100 & 95 & 60 & 5 & 10 & 15 & 25 & 35 & 45 & 55 & 70 & 80 & 90 \\
$Q_{9}$ & 100 & 95 & 90 & 65 & 5 & 10 & 20 & 30 & 40 & 50 & 60 & 75 & 85 \\
$Q_{10}$ & 100 & 95 & 90 & 85 & 70 & 5 & 15 & 25 & 35 & 45 & 55 & 65 & 80 \\
$Q_{11}$ & 100 & 95 & 90 & 85 & 80 & 75 & 10 & 20 & 30 & 40 & 50 & 60 & 70 \\
$Q_{12}$ & 100 & 95 & 90 & 85 & 80 & 75 & 70 & 10 & 20 & 30 & 40 & 50 & 60 \\
$Q_{13}$ & 100 & 95 & 90 & 85 & 80 & 75 & 70 & 60 & 10 & 20 & 30 & 40 & 50 \\
$Q_{14}$ & 100 & 95 & 90 & 85 & 80 & 75 & 70 & 60 & 50 & 10 & 20 & 30 & 40 \\
$Q_{15}$ & 100 & 95 & 90 & 85 & 80 & 75 & 70 & 60 & 50 & 40 & 10 & 20 & 30 \\
$Q_{16}$ & 100 & 95 & 90 & 85 & 80 & 75 & 70 & 60 & 50 & 40 & 30 & 10 & 20 \\
$Q_{17}$ & 100 & 95 & 90 & 85 & 80 & 75 & 70 & 60 & 50 & 40 & 30 & 20 & 10 \\
\hline
\end{tabular}


$j=5, \ldots, 17, z\left(Q_{k}, D_{j}\right)$ is the probability that buying $Q_{k}$ newspapers the newsboy will gain not less than $G\left(Q_{k}, D_{j}\right)$. Formally,

$$
z\left(Q_{k}, D_{j}\right)=\sum_{r: G\left(Q_{k}, D_{r}\right) \geq G\left(Q_{k}, D_{j}\right)} p_{r} .
$$

$z\left(Q_{k}, D_{j}\right)=p$ could be interpreted such that buying $Q_{k}$ newspapers the newsboy will gain not less than $G\left(Q_{k}, D_{j}\right)$ with probability $p$. In Table 2, we show the values of $z\left(Q_{k}, D_{j}\right)$, with $k, j=5, \ldots, 17$. Choosing to buy $Q_{k}$ newspapers, $k=5, \ldots, 17$, the best realization of the demand for the newsboy is $D_{j *}$ such that $z\left(Q_{k}, D_{j *}\right)=\min _{j \in\{5, \ldots, 17\}} z\left(Q_{k}, D_{j}\right)$.

After computing the values of $z\left(Q_{k}, D_{j}\right)$, for all $k, j=5, \ldots, 17$, for each quantile $\pi \in[0,1]$ one can define the following quantity:

$$
\rho\left(Q_{k}, \pi\right)=\max _{j: z\left(Q_{k}, D_{j}\right) \geq \pi}\left\{G\left(Q_{k}, D_{j}\right)\right\} .
$$

$\rho\left(Q_{k}, \pi\right)$ is the minimum gain the newsboy gets with probability at least equal to $\pi$ when buying $Q_{k}$ newspapers. The computation of $\rho\left(Q_{k}, \pi\right)$ can be explained in an easy way. Suppose the newsboy decides to buy 10 newspapers $\left(Q_{k}=10\right)$. We order the possible corresponding gains from the lowest to the greatest one as shown in Table 3 and, under the possible gains $G\left(Q_{10}, D_{j}\right)$, we show the corresponding probabilities $z\left(Q_{10}, D_{j}\right)$. After choosing, for example, the quantile $\pi=75 \%$, we look at the gains that could be obtained with probability no lower than $75 \%$, that are $1 €, 2 €, 3 €, 4 €$ and $4.95 €$, and we take the maximum among them, that is $4.95 €$. Obviously, in the extreme cases, choosing to buy 10 newspapers, the newsboy will gain with probability $99 \%$ at least $1 €\left(\rho\left(Q_{10}, 99 \%\right)=1 €\right)$ and with probability of the $1 \%$, at least $6 €$ $\left(\rho\left(Q_{10}, 1 \%\right)=6 €\right)$.

Table 3: Practical explanation of the computation of $\rho\left(Q_{10}, 75 \%\right)$

\begin{tabular}{c|cccccc|cccccccc}
\hline & $D_{5}$ & $D_{6}$ & $D_{7}$ & $D_{8}$ & $D_{17}$ & $D_{9}$ & $D_{16}$ & $D_{15}$ & $D_{14}$ & $D_{13}$ & $D_{12}$ & $D_{11}$ & $D_{10}$ \\
\hline$G\left(Q_{10}, D_{j}\right)$ & 1 & 2 & 3 & 4 & 4.95 & 5 & 5.10 & 5.25 & 5.40 & 5.55 & 5.70 & 5.85 & 6 \\
$z\left(Q_{10}, D_{j}\right)$ & 100 & 95 & 90 & 85 & 80 & 70 & 65 & 55 & 45 & 35 & 25 & 15 & 5 \\
\hline
\end{tabular}

In Table 4 we show the values of $\rho\left(Q_{k}, \pi\right)$, with $k=5, \ldots, 17$, corresponding to the meaningful quantiles for the $\mathrm{DM}\{1 \%, 25 \%, 50 \%, 75 \%, 99 \%\}$.

5.1 GRIP applied to the newsvendor problem

To help the newsboy to find the best number of newspapers to order, at first we will use the GRIP method; the newsboy can compare reference alternatives 
Table 4: Values of $\rho\left(Q_{k}, \pi\right)$, with $k=5, \ldots, 17$, corresponding to the meaningful quantiles for the DM $\{1 \%, 25 \%, 50 \%, 75 \%, 99 \%\}$

\begin{tabular}{|c|ccccc|}
\hline$\rho\left(Q_{k}, \pi\right)$ & $1 \%$ & $25 \%$ & $50 \%$ & $75 \%$ & $99 \%$ \\
\hline$Q_{5}$ & 3.00 & 2.40 & 1.95 & 1.50 & 1.20 \\
$Q_{6}$ & 3.60 & 3.00 & 2.60 & 2.25 & 1.95 \\
$Q_{7}$ & 4.20 & 3.60 & 3.30 & 3.00 & 2.20 \\
$Q_{8}$ & 4.80 & 4.35 & 3.90 & 3.60 & 1.80 \\
$Q_{9}$ & 5.40 & 4.95 & 4.65 & 4.35 & 1.40 \\
$Q_{10}$ & 6.00 & 5.70 & 5.25 & 4.95 & 1.00 \\
$Q_{11}$ & 6.60 & 6.30 & 6.00 & 5.60 & 0.60 \\
$Q_{12}$ & 7.20 & 6.90 & 6.60 & 5.20 & 0.20 \\
$Q_{13}$ & 7.80 & 7.50 & 7.20 & 4.80 & -0.20 \\
$Q_{14}$ & 8.40 & 8.10 & 7.40 & 4.40 & -0.60 \\
$Q_{15}$ & 9.00 & 8.70 & 7.00 & 4.00 & -1.00 \\
$Q_{16}$ & 9.60 & 8.60 & 6.60 & 3.60 & -1.40 \\
$Q_{17}$ & 10.20 & 8.20 & 6.20 & 3.20 & -1.80 \\
\hline
\end{tabular}

at comprehensive level, i.e. on all criteria, and at the partial level, i.e. on particular criteria.

Without any further information, the only information stemming from the values in Table 4 is the dominance relation $\Delta$ on the set of alternatives (in our case the different numbers of newspapers that could be bought from the newsboy) shown in Figure 1.

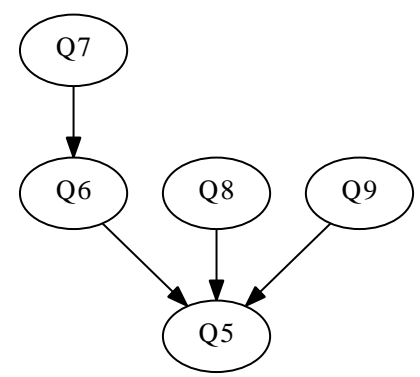

Fig. 1: Dominance relation on the set of considered alternatives

Let us suppose that the newsboy is confident on expressing four preferences regarding the alternatives $Q_{6}, Q_{7}, Q_{12}$ and $Q_{17}$; at first he says that, the difference between the evaluations of $Q_{12}$ and $Q_{6}$ with respect to quan- 
tile $75 \%$ is greater than the difference between the evaluations of $Q_{6}$ and $Q_{12}$ with respect to quantile $99 \%$. This piece of preference information is translated to the constraints $u_{75 \%}\left(Q_{12}\right)-u_{75 \%}\left(Q_{6}\right)>u_{99 \%}\left(Q_{6}\right)-u_{99 \%}\left(Q_{12}\right)$ and $u_{99 \%}\left(Q_{6}\right)-u_{99 \%}\left(Q_{12}\right)>0$. Including this piece of preference information, the corresponding necessary preference relation is shown in Figure 2. Bold arrows in Figure 2 represent new pairs in the necessary preference relation resulting from the first piece of preference information provided by the DM. Therefore, $Q_{11}$ and $Q_{12}$ are necessarily preferred to $Q_{6}$.

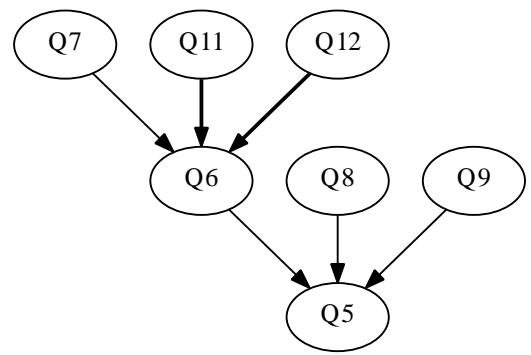

Fig. 2: Necessary preference relation obtained after including the first piece of preference information provided by the DM. Bold arrows represent new pairs of alternatives in the necessary preference relation

After that, the newsboy gives another piece of preference information at the partial level. Supposing to buy 7 newspapers, the newsboy prefers gaining at least $3 €$ with probability $75 \%$ than $2.20 €$ with probability $99 \%$, showing, therefore, to be quite risk prone. The constraint translating this piece of preference information is $u_{75 \%}\left(Q_{7}\right)>u_{99 \%}\left(Q_{7}\right)$. In Figure 3, we show the new necessary preference relation obtained after including the second piece of preference information provided by the DM. As one can observe, in consequence of this piece of information, six new pairs appear in the new necessary preference relation with respect to that one obtained in the previous step. In particular, $Q_{10}, Q_{13}, Q_{14}, Q_{15}, Q_{16}$ and $Q_{17}$ are now necessarily preferred to $Q_{5}$.

In the end, the newsboy provides the third piece of preference information stating, on one hand, that $Q_{17}$ is preferred to $Q_{7}$ and, on the other hand, that $Q_{12}$ is preferred to $Q_{17}$ more than $Q_{7}$ is preferred to $Q_{6}$. The constraints translating this piece of preference information are $U\left(Q_{17}\right)>U\left(Q_{7}\right)$ and $U\left(Q_{12}\right)-U\left(Q_{17}\right)>U\left(Q_{7}\right)-U\left(Q_{6}\right)$. The new necessary preference relation is shown in Figure 4.

Since not all alternatives are pairwise comparable in Figure 4 and the newsboy would like to have a complete ranking of the alternatives at hand, we compute a representative value function summarizing the ROR results. The marginal utilities and the comprehensive utility of each alternative are shown in Table 5. Looking at results in Table 5 it appears that the best choice for the newsboy is buying 12 newspapers, followed by the possibility of buying 


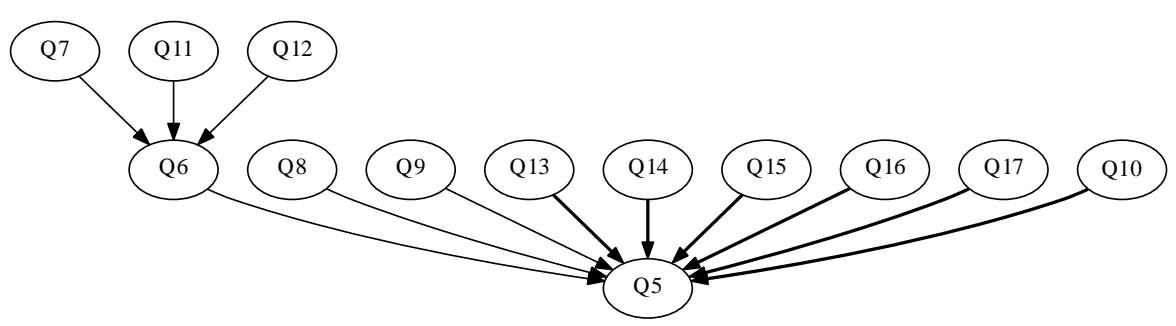

Fig. 3: Necessary preference relation obtained after including the second piece of preference information provided by the DM. Bold arrows represent new pairs of alternatives in the necessary preference relation

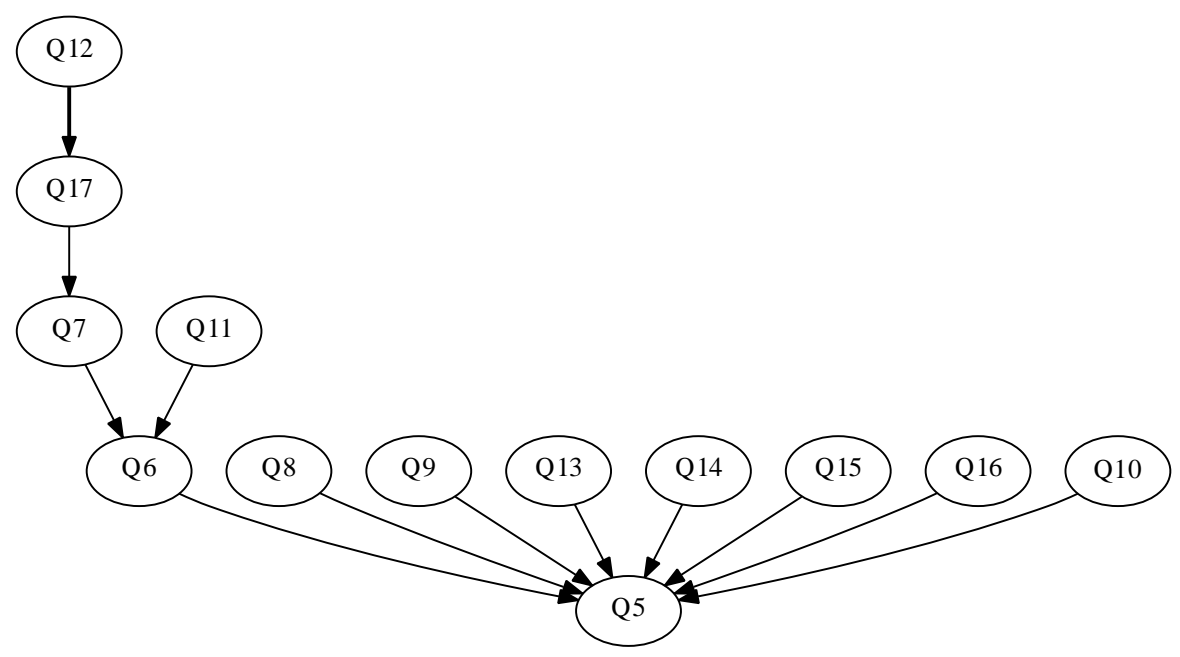

Fig. 4: Necessary preference relation obtained after including the last piece of preference information provided by the DM. Bold arrow represents the new pair of alternatives in the necessary preference relation

11 newspapers, and so on. Analogously, the worst choice for the newsboy is buying 5, 6 and 7 newspapers, being the alternatives presenting the lowest comprehensive utilities.

\subsection{ELECTRE ${ }^{\text {GKMS }}$ applied to the newsvendor problem}

In this subsection, we shall show how to apply the ELECTRE ${ }^{G K M S}$ method to obtain robust recommendations on the same problem we dealt with GRIP, on the base of the preference information provided by the DM; for the sake of simplicity, we shall suppose that the newsboy is able to provide the indifference, 
Table 5: Marginal Utilities with respect to the five quantiles and comprehensive utility of the alternatives at hand obtained by applying a representative value function

\begin{tabular}{|c|ccccc|c|}
\hline & $u_{1 \%}$ & $u_{25 \%}$ & $u_{50 \%}$ & $u_{75 \%}$ & $u_{99 \%}$ & $U$ \\
\hline$Q_{5}$ & 0 & 0 & 0 & 0 & $\sim 0$ & $\sim \mathbf{0}$ \\
$Q_{6}$ & 0.0384 & 0.0384 & 0.0368 & 0.0864 & $\sim 0$ & $\sim \mathbf{0 . 2}$ \\
$Q_{7}$ & 0.0647 & 0.0647 & 0.0619 & 0.2087 & $\sim 0$ & $\sim \mathbf{0 . 4}$ \\
$Q_{8}$ & 0.0966 & 0.0966 & 0.0914 & 0.2487 & $\sim 0$ & $\sim \mathbf{0 . 5 3 3 3}$ \\
$Q_{9}$ & 0.0966 & 0.0966 & 0.0914 & 0.2487 & $\sim 0$ & $\sim \mathbf{0 . 5 3 3 3}$ \\
$Q_{10}$ & 0.0966 & 0.0966 & 0.0914 & 0.3522 & $\sim 0$ & $\sim \mathbf{0 . 6 3 6 8}$ \\
$Q_{11}$ & 0.0966 & 0.0966 & 0.0914 & 0.5821 & $\sim 0$ & $\sim \mathbf{0 . 8 6 6 7}$ \\
$Q_{12}$ & 0.1289 & 0.1289 & 0.1601 & 0.5821 & $\sim 0$ & $\sim \mathbf{1}$ \\
$Q_{13}$ & 0.1289 & 0.1289 & 0.1601 & 0.2487 & $\sim 0$ & $\sim \mathbf{0 . 6 6 6 7}$ \\
$Q_{14}$ & 0.1289 & 0.1289 & 0.1601 & 0.2487 & $\sim 0$ & $\sim \mathbf{0 . 6 6 6 7}$ \\
$Q_{15}$ & 0.1289 & 0.1289 & 0.1601 & 0.2487 & $\sim 0$ & $\sim \mathbf{0 . 6 6 6 7}$ \\
$Q_{16}$ & 0.1289 & 0.1289 & 0.1601 & 0.2487 & $\sim 0$ & $\sim \mathbf{0 . 6 6 6 7}$ \\
$Q_{17}$ & 0.1289 & 0.1289 & 0.1190 & 0.2232 & 0 & $\sim \mathbf{0 . 6}$ \\
\hline
\end{tabular}

preference and veto thresholds shown in Table 6 . As a consequence, in this problem, the unknown parameters are the cutting level $\lambda$ and the weights underlining the importance assigned to the considered quantiles.

Table 6: Thresholds on $\rho\left(Q_{k}, \pi\right)$ provided by the DM for the considered quantiles

\begin{tabular}{|c|cccc|}
\hline & $q_{\pi}$ & $p_{\pi}$ & $v_{\pi}$ \\
\hline$\pi=1 \%$ & 1.0 & 2.0 & 4.0 \\
$\pi=25 \%$ & 1.0 & 2.0 & 4.0 \\
$\pi=50 \%$ & 0.5 & 1.5 & 3.0 \\
$\pi=75 \%$ & 0.5 & 1.5 & 3.0 \\
$\pi=99 \%$ & 0.5 & 1.5 & 3.0 \\
\hline
\end{tabular}

Let's suppose that the newsboy states that $Q_{12}$ outranks $Q_{6}\left(Q_{12} S Q_{6}\right)$ and, at the same time, $Q_{5}$ does not outrank $Q_{8}\left(Q_{5} S^{C} Q_{8}\right)$. By solving the programming problem presented in Appendix A, there exists at least one instance of the preference model compatible with the preference provided by the DM. On the basis of this preference information, we obtained the necessary and possible outranking relations shown in Tables 7 and 8 . 
Table 7: Necessary outranking relation for the ELECTRE ${ }^{G K M S}$ method computed on the base of the preference information provided by the DM

\begin{tabular}{|c|cccccccccccccc|}
\hline & $Q_{5}$ & $Q_{6}$ & $Q_{7}$ & $Q_{8}$ & $Q_{9}$ & $Q_{10}$ & $Q_{11}$ & $Q_{12}$ & $Q_{13}$ & $Q_{14}$ & $Q_{15}$ & $Q_{16}$ & $Q_{17}$ \\
\hline$Q_{5}$ & 1 & 0 & 0 & 0 & 0 & 0 & 0 & 0 & 0 & 0 & 0 & 0 & 0 \\
$Q_{6}$ & 1 & 1 & 0 & 0 & 0 & 0 & 0 & 0 & 0 & 0 & 0 & 0 & 0 \\
$Q_{7}$ & 1 & 1 & 1 & 0 & 0 & 0 & 0 & 0 & 0 & 0 & 0 & 0 & 0 \\
$Q_{8}$ & 1 & 1 & 1 & 1 & 0 & 0 & 0 & 0 & 0 & 0 & 0 & 0 & 0 \\
$Q_{9}$ & 1 & 1 & 1 & 1 & 1 & 0 & 0 & 0 & 0 & 0 & 0 & 0 & 0 \\
$Q_{10}$ & 1 & 1 & 1 & 1 & 1 & 1 & 0 & 0 & 0 & 0 & 0 & 0 & 0 \\
$Q_{11}$ & 1 & 1 & 1 & 1 & 1 & 1 & 1 & 0 & 0 & 0 & 0 & 0 & 0 \\
$Q_{12}$ & 1 & 1 & 1 & 1 & 1 & 1 & 1 & 1 & 0 & 0 & 0 & 0 & 0 \\
$Q_{13}$ & 1 & 1 & 1 & 1 & 1 & 1 & 0 & 1 & 1 & 1 & 0 & 0 & 0 \\
$Q_{14}$ & 1 & 1 & 1 & 1 & 1 & 0 & 0 & 0 & 1 & 1 & 1 & 0 & 0 \\
$Q_{15}$ & 1 & 1 & 0 & 1 & 1 & 0 & 0 & 0 & 0 & 1 & 1 & 1 & 0 \\
$Q_{16}$ & 1 & 0 & 0 & 0 & 0 & 0 & 0 & 0 & 0 & 0 & 1 & 1 & 1 \\
$Q_{17}$ & 0 & 0 & 0 & 0 & 0 & 0 & 0 & 0 & 0 & 0 & 0 & 1 & 1 \\
\hline
\end{tabular}

Table 8: Possible outranking relation for the ELECTRE ${ }^{G K M S}$ method computed on the base of the preference information provided by the DM

\begin{tabular}{|c|cccccccccccccc|}
\hline & $Q_{5}$ & $Q_{6}$ & $Q_{7}$ & $Q_{8}$ & $Q_{9}$ & $Q_{10}$ & $Q_{11}$ & $Q_{12}$ & $Q_{13}$ & $Q_{14}$ & $Q_{15}$ & $Q_{16}$ & $Q_{17}$ \\
\hline$Q_{5}$ & 1 & 1 & 1 & 1 & 0 & 0 & 0 & 0 & 0 & 0 & 0 & 0 & 0 \\
$Q_{6}$ & 1 & 1 & 1 & 1 & 1 & 0 & 0 & 0 & 0 & 0 & 0 & 0 & 0 \\
$Q_{7}$ & 1 & 1 & 1 & 1 & 1 & 1 & 0 & 0 & 0 & 0 & 0 & 0 & 0 \\
$Q_{8}$ & 1 & 1 & 1 & 1 & 1 & 1 & 1 & 0 & 0 & 0 & 0 & 0 & 0 \\
$Q_{9}$ & 1 & 1 & 1 & 1 & 1 & 1 & 1 & 1 & 1 & 1 & 1 & 0 & 0 \\
$Q_{10}$ & 1 & 1 & 1 & 1 & 1 & 1 & 1 & 1 & 1 & 1 & 1 & 1 & 0 \\
$Q_{11}$ & 1 & 1 & 1 & 1 & 1 & 1 & 1 & 1 & 1 & 1 & 1 & 1 & 1 \\
$Q_{12}$ & 1 & 1 & 1 & 1 & 1 & 1 & 1 & 1 & 1 & 1 & 1 & 1 & 1 \\
$Q_{13}$ & 1 & 1 & 1 & 1 & 1 & 1 & 1 & 1 & 1 & 1 & 1 & 1 & 1 \\
$Q_{14}$ & 1 & 1 & 1 & 1 & 1 & 1 & 1 & 1 & 1 & 1 & 1 & 1 & 1 \\
$Q_{15}$ & 1 & 1 & 0 & 1 & 1 & 1 & 1 & 1 & 1 & 1 & 1 & 1 & 1 \\
$Q_{16}$ & 1 & 0 & 0 & 0 & 1 & 1 & 1 & 1 & 1 & 1 & 1 & 1 & 1 \\
$Q_{17}$ & 0 & 0 & 0 & 0 & 0 & 1 & 1 & 1 & 1 & 1 & 1 & 1 & 1 \\
\hline
\end{tabular}


To summarize the results got by the application of the ROR in this case, we use the same procedure presented in [29]. On the base of the results shown in Tables 7 and 8 , and reminding that $a S^{C P} b$ iff $\operatorname{not}\left(a S^{N} b\right)$, and $a S^{C N} b$ iff $\operatorname{not}\left(a S^{P} b\right)$, for each alternative $Q_{k}, k=5, \ldots, 17$, we computed the following values, where $A=\left\{Q_{k}, k=5, \ldots, 17\right\}$ :

- $T_{S^{N}}\left(Q_{k}\right)=\left|Q_{r} \in A, r \neq k: Q_{k} S^{N} Q_{r}\right|$; that is the number of alternatives $Q_{r} \in A$ such that $Q_{k}$ necessarily outranks $Q_{r}$,

- $W_{S^{N}}\left(Q_{k}\right)=\left|Q_{r} \in A, r \neq k: Q_{r} S^{N} Q_{k}\right|$; that is the number of alternatives $Q_{r} \in A$ such that $Q_{r}$ necessarily outranks $Q_{k}$,

- $T_{S^{C N}}\left(Q_{k}\right)=\left|Q_{r} \in A: \operatorname{not}\left(Q_{r} S^{P} Q_{k}\right)\right|$; that is the number of alternatives $Q_{r} \in A$ such that $Q_{r}$ does not possibly outrank $Q_{k}$,

- $W_{S^{C N}}\left(Q_{k}\right)=\left|Q_{r} \in A: \operatorname{not}\left(Q_{k} S^{P} Q_{r}\right)\right|$; that is the number of alternatives $Q_{r} \in A$ such that $Q_{k}$ does not possibly outrank $Q_{r}$,

- $T\left(Q_{k}\right)=T_{S^{N}}\left(Q_{k}\right)+T_{S^{C N}}\left(Q_{k}\right), W\left(Q_{k}\right)=W_{S^{N}}\left(Q_{k}\right)+W_{S^{C N}}\left(Q_{k}\right)$ and $N F S\left(Q_{k}\right)=T\left(Q_{k}\right)-W\left(Q_{k}\right)$

Table 9: Values summarizing the results of the necessary and possible outranking relations provided in Tables 7 and 8

\begin{tabular}{|c|cc|cc|cc|c|}
\hline & $T_{S^{N}}(Q)$ & $W_{S^{N}}(Q)$ & $T_{S^{C N}}(Q)$ & $W_{S^{C N}}(Q)$ & $T(Q)$ & $W(Q)$ & $N F S(Q)$ \\
\hline$Q_{5}$ & 0 & 11 & 1 & 9 & 1 & 20 & -19 \\
$Q_{6}$ & 1 & 9 & 2 & 8 & 3 & 17 & -14 \\
$Q_{7}$ & 2 & 7 & 3 & 7 & 5 & 14 & -9 \\
$Q_{8}$ & 3 & 7 & 2 & 6 & 5 & 13 & -8 \\
$Q_{9}$ & 4 & 6 & 2 & 2 & 6 & 8 & -2 \\
$Q_{10}$ & 5 & 3 & 2 & 1 & 7 & 4 & 3 \\
$Q_{11}$ & 6 & 1 & 3 & 0 & 9 & 1 & 8 \\
$Q_{12}$ & 7 & 1 & 4 & 0 & 11 & 1 & 10 \\
$Q_{13}$ & 8 & 1 & 4 & 0 & 12 & 1 & 11 \\
$Q_{14}$ & 7 & 2 & 4 & 0 & 11 & 2 & 9 \\
$Q_{15}$ & 6 & 2 & 4 & 1 & 10 & 3 & 7 \\
$Q_{16}$ & 3 & 2 & 5 & 3 & 8 & 5 & 3 \\
$Q_{17}$ & 1 & 1 & 6 & 5 & 7 & 6 & 1 \\
\hline
\end{tabular}

By using the values of the Net Flow Score (NFS) shown in Table 9, we obtain the following complete ranking of the alternatives at hand:

$Q_{13} \succ Q_{12} \succ Q_{14} \succ Q_{11} \succ Q_{15} \succ Q_{16} \sim Q_{10} \succ Q_{17} \succ Q_{9} \succ Q_{8} \succ Q_{7} \succ Q_{6} \succ Q_{5}$.

In this case, one can see that the best choice for the newsboy is buying 13 newspapers while, the worst one, is buying 5 newspapers only. As one can see, 
while both methods (GRIP and ELECTRE ${ }^{\text {GKMS }}$ ) recommend that buying 5 newspapers is the worst choice for the newsboy, the recommendation on the best choice is different on the two methods (12 newspapers is the best choice if one applies GRIP, while 13 is the best choice if one decides to use ELECTRE $^{G K M S}$ ). This is not surprising since GRIP, as all additive methods, is a compensatory method while ELECTRE ${ }^{G K M S}$, as all outranking methods, is not compensatory.

\section{Conclusions}

In this paper we presented a new methodology to handle decision under risk and uncertainty in a constructive approach. This methodology is based on two points:

- On one hand, the risk and the uncertainty is represented through meaningful quantiles. They are very manageable, since they permit to deal with additive and nonadditive probabilities as well as with qualitative probabilities. Quantiles are very understandable for the DM because, taking into account the limitation of the human mind, few quantiles can synthesize all the very often rich and complex information contained in a probability distribution.

- On the other hand, ROR is used to induce in a cautious way new preferences from the whole set of instances of the adopted preference model compatible with the preference information supplied by the DM.

We have shown in an illustrative example how the proposed methodology can be applied to a very classical problem of operational research, namely the newsvendor problem. We believe that the proposed methodology has quite good properties and that the results shown by the illustrative example are convincing. This encourages to further develop this approach in a number of directions that we sketch in the following:

- in this paper we considered application of ROR to additive multiple attribute value functions and ELECTRE approach, but there are other MCDA methods to which ROR has been applied and that can be used to handle decision under risk and uncertainty, such as PROMETHEE methods [39], Choquet integral [1] and an enriched multiattribute value function [34];

- our methodology could be applied also to Stochastic Ordinal Regression (SOR) $[41,42]$ which has been recently proposed, coupling ROR with Stochastic Multicriteria Acceptability Analysis (SMAA) [46] in which a probability distribution is considered on the family of all compatible instances of the preference model to determine the probability that alternative $a$ is preferred to alternative $b$, or that alternative $a$ ranks in the $k$-th position;

- ROR has been recently coupled with Evolutionary Multiobjective Optimization (EMO) [16], to focus the research of the nondominated solutions in the part of Pareto front that is the most preferred by the DM [5,7]; our methodology of decision under risk and uncertainty can also be applied to this class of EMO algorithms based on ROR; 
- in this paper we considered the newsvendor problem, however, there are many other problems in operational research formulated in terms of decision under risk and uncertainty; only to give another classical example, let us mention the Markowitz's financial portfolio choice problem [49], that has been already treated in terms of quantiles but considering ordinal regression rather than ROR in [30];

- in this paper we considered risk and uncertainty in monocriterion problems that were transformed in multicriteria problems using quantiles; however, we can apply our methodology also in the context of risk and uncertainty related to a plurality of criteria, and for each criterion we can consider a certain number of quantiles;

- one can consider not only the aspect of risk and uncertainty, but also the aspect of distribution of the consequences of a decision over time, which is a common framework in which practically all real world problems are formulated. Our approach can be extended to take into account also this aspect.

In conclusion, we believe that, since our approach permits to deal in an effective way with the risk and uncertainty aspects present in all real world decision problems, it can be considered as an important component of the toolboox of operational research.

Acknowledgements The first two authors wish to acknowledge funding by the "Programma Operativo Nazionale" Ricerca \& Competitivitá "2007-2013" within the project "PON04a2 E SINERGREEN-RES-NOVAE".

The fourth author wishes to acknowledge financial support from the Polish National Science Center, DEC-2015/18/A/ST6/00171.

\section{References}

1. S. Angilella, S. Greco, and B. Matarazzo. Non-additive robust ordinal regression: A multiple criteria decision model based on the Choquet integral. European Journal of Operational Research, 201(1):277-288, 2010.

2. F.J. Anscombe and R.J. Aumann. A Definition of Subjective Probability. Annals of Mathematical Statistics, pages 199-205, 1963.

3. D.E Bell, H. Raiffa, and A. Tversky. Decision making: Descriptive, normative, and prescriptive interactions. Cambridge: Cambridge University Press, 1988

4. T. Bewley. Knightian decision theory: part I. Decisions in Economics and Finance, 25:79-110, 2002.

5. J. Branke, S. Corrente, S. Greco, R. Słowiński and P. Zielniewicz. Using Choquet integral as preference model in interactive evolutionary multiobjective optimization European Journal of Operational Research. DOI:10.1016/j.ejor.2015.10.027.

6. J. Branke, K. Deb, K. Miettinen, and R. Słowiński. Multiobjective Optimization. LNCS, vol. 5252. Springer, Heidelberg, 2008.

7. J. Branke, S. Greco, R. Słowiński, and P. Zielniewicz. Learning value functions in interactive evolutionary multiobjective optimization. Evolutionary Computation, IEEE Transactions on, 19(1):88-102, 2015.

8. J.P. Brans and Ph. Vincke. A preference ranking organisation method: The PROMETHEE method for MCDM. Management Science, 31(6):647-656, 1985.

9. C.P. Chambers. Ordinal aggregation and quantiles. Journal of Economic Theory, 137(1):416-431, 2007. 
10. G. Choquet. Theory of capacities. Annales de l'Institut Fourier, 5:131-295, 1953.

11. S. Corrente, S. Greco, and A. Ishizaka. Combining Analytical Hierarchy Process and Choquet integral within non-additive robust ordinal regression. Omega. DOI:10.1016/j.omega.2015.07.003.

12. S. Corrente, S. Greco, M. Kadziński, and R. Słowiński. Robust ordinal regression in preference learning and ranking. Machine Learning, 93:381-422, 2013.

13. S. Corrente, S. Greco, M. Kadziński, and R. Słowiński. Robust Ordinal Regression. Wiley Encyclopedia of Operations Research and Management Science, pages 1-10, 2014.

14. S. Corrente, S. Greco, and R. Słowiński. Multiple Criteria Hierarchy Process in Robust Ordinal Regression. Decision Support Systems, 53(3):660-674, 2012.

15. S. Corrente, S. Greco, and R. Słowiński. Multiple Criteria Hierarchy Process with ELECTRE and PROMETHEE. Omega, 41:820-846, 2012.

16. K. Deb. Multi-objective optimization using evolutionary algorithms, Volume 16. John Wiley \& Sons, 2001.

17. L. Dias and V. Mousseau. Inferring ELECTREs veto-related parameters from outranking examples. European Journal of Operational Research, 170(1):172-191, 2006.

18. F.Y. Edgeworth. The mathematical theory of banking. Journal of the Royal Statistical Society, 51(1):113-127, 1888.

19. D. Ellsberg. Risk, ambiguity, and the Savage axioms. The quarterly journal of economics, pages 643-669, 1961.

20. J. Figueira, S. Greco, and M. Ehrgott. Multiple Criteria Decision Analysis: State of the Art Surveys. Springer, Berlin, 2005.

21. J. Figueira, S. Greco, and R. Słowiński. Building a set of additive value functions representing a reference preorder and intensities of preference: GRIP method. European Journal of Operational Research, 195(2):460-486, 2009.

22. J. Figueira, S. Greco, and R. Słowiński. Identifying the "most representative" value function among all compatible value functions in the GRIP. Proceedings of the 68th EURO Working Group on MCDA, Chania, October 2008.

23. J. Figueira, S. Greco, and R. Słowiński. Building a set of additive value functions representing a reference preorder and intensities of preference: GRIP method. European Journal of Operational research, 195(2):460-486, 2009.

24. J. R. Figueira, S. Greco, R. Słowiński, and B. Roy. An overview of ELECTRE methods and their recent extensions. Journal of Multi-Criteria Decision Analysis, 20:61-85, 2013.

25. J. Figueira, V. Mousseau, and B. Roy. ELECTRE methods. Multiple Criteria Decision Analysis: State of the Art Surveys, pages 133-153, 2005.

26. A. Giarlotta and S. Greco. Necessary and possible preference structures. Journal of Mathematical Economics, 49(2):163-172, 2013.

27. I. Gilboa, F. Maccheroni, M. Marinacci, and D. Schmeidler. Objective and subjective rationality in a multiple prior model. Econometrica, 78(2):755-770, 2010.

28. I. Gilboa and D. Schmeidler. Maxmin expected utility with non-unique prior. Journal of mathematical economics, 18(2):141-153, 1989.

29. S. Greco, M. Kadziński, V.Mousseau, and R. Słowiński. ELECTREGKMS : Robust ordinal regression for outranking methods. European Journal of Operational Research, 214(1):118-135, 2011.

30. S. Greco, B. Matarazzo, and R. Slowinski. Beyond Markowitz with multiple criteria decision aiding. Journal of Business Economics, 83(1):29-60, 2013.

31. S. Greco, B. Matarazzo, and R. Słowiński. Dominance-based rough set approach to decision under uncertainty and time preference. Annals of Operations Research, 176(1):4175,2010 .

32. S. Greco, B. Matarazzo, and R. Słowiński. Rough sets theory for multicriteria decision analysis. European Journal of Operational Research, 129(1):1-47, 2001.

33. S. Greco, V. Mousseau, and R. Słowiński. Ordinal regression revisited: multiple criteria ranking using a set of additive value functions. European Journal of Operational Research, 191(2):415-435, 2008.

34. S. Greco, V. Mousseau, and R. Słowiński. Robust ordinal regression for value functions handling interacting criteria. European Journal of Operational Research, 239(3):711-730, 2014.

35. J.S. Hammond, R.L. Keeney, and H. Raiffa. Smart choices: A practical guide to making better decisions, volume 226. Harvard Business Press, 1999. 
36. E. Jacquet-Lagrèze and J. Siskos. Assessing a set of additive utility functions for multicriteria decision-making, the UTA method. European Journal of Operational Research, 10(2):151-164, 1982.

37. E. Jacquet-Lagrèze and Y. Siskos. Preference disaggregation: 20 years of MCDA experience. European Journal of Operational Research, 130(2):233-245, 2001.

38. P. Jorion. Value at risk: the new benchmark for managing financial risk (3rd ed.). McGraw-Hill, 2007.

39. M. Kadziński, S. Greco, and R. Słowiński. Extreme ranking analysis in robust ordinal regression. Omega, 40(4):488-501, 2012.

40. M. Kadziński, S. Greco, and R. Słowiński. Selection of a representative value function in robust multiple criteria ranking and choice. European Journal of Operational Research, 217(3):541-553, 2011.

41. M. Kadziński and T. Tervonen. Robust multi-criteria ranking with additive value models and holistic pair-wise preference statements. European Journal of Operational Research, 228(1):169-180, 2013.

42. M. Kadziński and T. Tervonen. Stochastic ordinal regression for multiple criteria sorting problems. Decision Support Systems, 55(11):55-66, 2013.

43. R.L. Keeney and H. Raiffa. Decisions with multiple objectives: Preferences and value tradeoffs. J. Wiley, New York, 1993.

44. B. Khouja. The single-period (news-vendor) problem: literature review and suggestions for future research. Omega, 27(5):537-553, 1999.

45. F.H. Knight. Risk, uncertainty and profit. New York: Harper \& Row 1921.

46. R. Lahdelma, J. Hokkanen, and P. Salminen. SMAA - stochastic multiobjective acceptability analysis. European Journal of Operational Research, 106(1):137-143, 1998.

47. R.N. Langlois and M.M. Cosgel. Frank Knight on risk, uncertainty, and the firm: a new interpretation. Economic Inquiry, 31(3):456-465, 1993.

48. C.F. Manski. Ordinal utility models of decision making under uncertainty. Theory and Decision, 25(1):79-104, 1988.

49. H. Markowitz. Portfolio selection. The Journal of Finance, 7(1):77-91, 1952.

50. M.A. Matos. Decision under risk as a multicriteria problem. European Journal of Operational Research, 181(3):1516-1529, 2007.

51. G.A. Miller. The magical number seven, plus or minus two: some limits on our capacity for processing information. Psychological review, 63(2):81, 1956.

52. V. Mousseau and L. Dias. Valued outranking relations in ELECTRE providing manageable disaggregation procedures. European Journal of Operational Research, 156(2):467482, 2004.

53. V. Mousseau, J.R. Figueira, L. Dias, C. Gomes da Silva, and J. Climaco. Resolving inconsistencies among constraints on the parameters of an MCDA model. European Journal of Operational Research, 147(1):72-93, 2003.

54. N. Petruzzi, and M. Dada. Pricing and the newsvendor problem: A review with extensions. Operations Research, 47(2):183-194, 1999.

55. Y. Qin, R. Wang, A.J. Vakharia, Y. Chen and M.M.H. Seref. The newsvendor problem: Review and directions for future research. European Journal of Operational Research, 213(2):361-374,2011.

56. M. Rostek. Quantile maximization in decision theory. The Review of Economic Studies, $77(1): 339-371,2010$.

57. B. Roy. Decision science or decision-aid science? European Journal of Operational Research, 66(2):184-203, 1993

58. B. Roy. Multicriteria Methodology for Decision Aiding. Kluwer, Dordrecht, 1996.

59. J.E. Russo and P.J.H Schoemaker. Decision traps: Ten barriers to brilliant decisionmaking and how to overcome them. Doubleday Publishing Co., New York, 1989.

60. L.J. Savage. The foundations of statistics. New York: Wiley, 1954.

61. D. Schmeidler. Subjective probability and expected utility without additivity. Econometrica: Journal of the Econometric Society, 57:571-587, 1989.

62. R. Słowiński, S. Greco, and B. Matarazzo. Rough sets in decision making. In R.A. Meyers, editor, Encyclopedia of Complexity and Systems Science, pages 7753-7786, New York, 2009. Springer. 
63. R. Słowiński, S. Greco, and B. Matarazzo. Rough Set Methodology for Decision Aiding. Chapter 22. In J. Kacprzyk and W. Pedrycz, editors, Handbook of Computational Intelligence, pages 349-370, Berlin, 2015. Springer.

64. J. Von Neumann and O. Morgenstern. Theory of games and economic behavior. Princeton: Princeton University Press, 1944.

\section{Appendix A (Constraints of the ELECTRE ${ }^{G K M S}$ method)}

In this Appendix, we shall give more details on the technical constraints of the ELECTRE ${ }^{G K M S}$ method as well as on the constraints translating the preference information provided by the DM.

- Preference information on comparisons between alternatives $a^{*}, b^{*} \in A^{R} \subseteq$ $A$ :

- For all $\left(a^{*}, b^{*}\right) \in A^{R}$ such that $a^{*} S b^{*}$ :

$C\left(a^{*}, b^{*}\right)=\sum_{j=1}^{m} \psi_{j}\left(a^{*}, b^{*}\right) \geq \lambda$ and $g_{j}\left(b^{*}\right)-g_{j}\left(a^{*}\right)<v_{j}\left(a^{*}\right), j=1, \ldots, m$,

where $\psi_{j}(a, b)=\varphi_{j}(a, b) \times w_{j}$ for all $j$ and for all $(a, b) \in A \times A$.

- For all $\left(a^{*}, b^{*}\right) \in A^{R}$ such that $a^{*} S^{C} b^{*}$ :

$$
C\left(a^{*}, b^{*}\right)=\sum_{j=1}^{m} \psi_{j}\left(a^{*}, b^{*}\right)<\lambda \text { or } \exists j \in G: g_{j}\left(b^{*}\right)-g_{j}\left(a^{*}\right) \geq v_{j}\left(a^{*}\right),
$$

which can be modeled as:

$C\left(a^{*}, b^{*}\right)=\sum_{j=1}^{m} \psi_{j}\left(a^{*}, b^{*}\right)<\lambda+M_{0}\left(a^{*}, b^{*}\right)$ and $g_{j}\left(b^{*}\right)-g_{j}\left(a^{*}\right) \geq v_{j}\left(a^{*}\right)-\delta M_{j}\left(a^{*}, b^{*}\right)$,

where $M_{j}\left(a^{*}, b^{*}\right) \in\{0,1\}, j=0, \ldots, m, \sum_{j=0}^{m} M_{j}\left(a^{*}, b^{*}\right) \leq m$ and $\delta$ is an auxiliary variable equal to a big positive value (i.e. $\delta \geq \max _{j}\left\{x_{j}^{m_{j}}-\right.$ $\left.\left.x_{j}^{0}\right\}\right)$.

A vector $M$ of binary variables is used to express that either the concordance test or non-discordance test has to be negative. Notice that if $M_{j}\left(a^{*}, b^{*}\right)=0$, then a corresponding $j$-th condition causes discordance with statement $a^{*} S b^{*}$. On the contrary, if $M_{j}\left(a^{*}, b^{*}\right)=1$, then the respective condition is always satisfied, not making veto. However, there has to be at least one non-equality for which $M_{j}\left(a^{*}, b^{*}\right)=0$, as $\sum_{j=0}^{m} M_{j}\left(a^{*}, b^{*}\right) \leq m$. 
- Limitations on the values of inter-criteria parameters: $\lambda, v_{j}(a), \forall a \in A$, and $w_{j} j=1, \ldots, m$ :

- The range of allowed values of a concordance threshold, $0.5 \leq \lambda \leq 1.0$,

- Normalization of the marginal concordance indices for all criteria so that the indices corresponding to the largest difference in evaluations of two alternatives on each criterion $\left(g_{j}\left(a_{j}^{*}\right)-g_{j}\left(a_{j, *}\right)=x_{j}^{m_{j}}-x_{j}^{0}\right)$ sum up to 1:

$$
\sum_{j=1}^{m} \psi_{j}\left(a_{j}^{*}, a_{j, *}\right)=1 \text { with } a_{j}^{*}, a_{j, *} \in A, j=1, \ldots, m \text {. }
$$

As we previously normalized weights of the criteria so that they sum up to 1 , each weight is now understood as a maximal share of each criterion in the global concordance index. Consequently, $w_{j}=\psi_{j}\left(a_{j}^{*}, a_{j, *}\right), j=$ $1, \ldots, m$.

- Lower bounds on the values of veto thresholds $v_{j}(a), j=1, \ldots, m, \forall a \in$ A:

- $v_{j}(a)$ needs to be larger than corresponding preference threshold (the greatest value of a preference threshold $p_{j}^{R}(a)$ allowed by the $\mathrm{DM}), v_{j}(a)>p_{j}^{R}(a), j \in G_{1}^{2}$,

- $v_{j}(a)$ is required to be larger than $g_{j}(b)-g_{j}(a)$ for all pairs of alternatives $(a, b)$ for which the DM stated that the difference between $g_{j}(a)$ and $g_{j}(b)$ is non-significant $\left(g_{j}(a) \leq g_{j}(b)\right), a \sim_{j} b, j \in G_{2}{ }^{3}$ (we do not refer here to pairs $\left(a^{*}, b^{*}\right)$ such that $a^{*} \succ_{j} b^{*}$, because even if the DM states that the difference between $g_{j}\left(a^{*}\right)$ and $g_{j}\left(b^{*}\right)$ is relevant, the veto threshold $v_{j}$ can still be less, equal, or larger than $\left.g_{j}\left(a^{*}\right)-g_{j}\left(b^{*}\right)\right)$.

- The function determining the values of veto thresholds $v_{j}(a)$ is required to be monotone non-decreasing with respect to $g_{j}(a)$ :

$v_{j}(a) \geq v_{j}(b)$ if $g_{j}(a)>g_{j}(b)$ and $v_{j}(a)=v_{j}(b)$ if $g_{j}(a)=g_{j}(b), j=1, \ldots, m$.

If the DM wishes to model the veto threshold with a constant value (not dependent on $g_{j}(a)$ ), we would skip the monotonicity constraints and replace $v_{j}(a)$ with $v_{j}$ in all aforementioned formulas.

\footnotetext{
${ }^{2} G_{1}$ is the subset of criteria $G$ on which the DM has expressed direct preferences of the type 3 . and 4. shown in Section 4.2

$3 G_{2}$ is the subset of criteria $G$ on which the DM has expressed indirect preferences of the type 5 . and 6 . shown in Section 4.2
} 
- Restrictions concerning the value of marginal concordance indices $\psi_{j}(a, b), j=$ $1, \ldots, m$ :

$-\psi_{j}(a, b)=0$ if $g_{j}(b)-g_{j}(a) \geq p_{j}^{R}(a)$, for all $(a, b) \in A \times A$,

$-\psi_{j}(a, b)>0$ if $g_{j}(a)-g_{j}(b)>-p_{j}^{L}(a)$ for all $(a, b) \in A \times A$,

- $\psi_{j}(a, b)=\psi_{j}\left(a_{j}^{*}, a_{j, *}\right)$ if $g_{j}(a)-g_{j}(b) \geq-q_{j}^{L}(a)$, for all $(a, b) \in A \times A$;

$-\psi_{j}(a, b)<\psi_{j}\left(a_{j}^{*}, a_{j, *}\right)$ if $g_{j}(b)-g_{j}(a)>q_{j}^{R}(a)$, for all $(a, b) \in A \times A$,

$-\psi_{j}(a, b)=0$ if $b \succ_{j} a$,

$-\psi_{j}(a, b)=\psi_{j}\left(a_{j}^{*}, a_{j, *}\right), \psi_{j}(b, a)=\psi_{j}\left(a_{j}^{*}, a_{j, *}\right)$ if $a \sim_{j} b$.

- Monotonicity of the functions of marginal concordance indices $\psi_{j}(a, b), j=$ $1, \ldots, m$ :

- If, according to the preferences of the DM, indifference $q_{j}$ and preference $p_{j}$ thresholds for criterion $g_{j}$ are not dependent on $g_{j}(a)$, then $\forall a, b, c, d \in A$, and for $j=1, \ldots, m$ :

- $\psi_{j}(a, b) \geq \psi_{j}(c, d)$ if $g_{j}(a)-g_{j}(b)>g_{j}(c)-g_{j}(d)$,

- $\psi_{j}(a, b)=\psi_{j}(c, d)$ if $g_{j}(a)-g_{j}(b)=g_{j}(c)-g_{j}(d)$.

- If according to the preferences of the DM indifference $q_{j}(a)$ and preference $p_{j}(a)$ thresholds depend on the value of $g_{j}(a)$, then, for $j=$ $1, \ldots, m$ :

- $\psi_{j}(a, c) \geq \psi_{j}(b, c)$ if $g_{j}(a)>g_{j}(b)$ for all $a, b, c \in A$,

- $\psi_{j}(a, c)=\psi_{j}(b, c)$ if $g_{j}(a)=g_{j}(b)$ for all $a, b, c \in A$,

- $\psi_{j}(a, b) \geq \psi_{j}(a, c)$ if $g_{j}(b)<g_{j}(c)$ for all $a, b, c \in A$,

- $\psi_{j}(a, b)=\psi_{j}(a, c)$ if $g_{j}(b)=g_{j}(c)$ for all $a, b, c \in A$.

The whole set of monotonicity and normalization constraints together with the constraints translating the preference information provided by the DM is denoted by $E^{A^{R^{\prime}}}$ : 
Pairwise comparison stating $a^{*} S b^{*}$ or $a^{*} S^{C} b^{*}$ :

$$
\begin{aligned}
& C\left(a^{*}, b^{*}\right)=\sum_{j=1}^{m} \psi_{j}\left(a^{*}, b^{*}\right) \geq \lambda \text { and } g_{j}\left(b^{*}\right)-g_{j}\left(a^{*}\right)+\varepsilon \leq v_{j}\left(a^{*}\right), j=1, \ldots, m, \\
& \quad \text { if } a^{*} S b^{*}, \text { for }\left(a^{*}, b^{*}\right) \in A^{R}, \\
& C\left(a^{*}, b^{*}\right)=\sum_{j=1}^{m} \psi_{j}\left(a^{*}, b^{*}\right)+\varepsilon \leq \lambda+M_{0}\left(a^{*}, b^{*}\right) \text { and } g_{j}\left(b^{*}\right)-g_{j}\left(a^{*}\right) \geq v_{j}\left(a^{*}\right)-\delta M_{j}\left(a^{*}, b^{*}\right), \\
& \quad \text { if } a^{*} S^{C} b^{*}, \text { for }\left(a^{*}, b^{*}\right) \in A^{R}, \\
& \quad M_{j}\left(a^{*}, b^{*}\right) \in\{0,1\}, j=0, \ldots, m, \sum_{j=0}^{m} M_{j}\left(a^{*}, b^{*}\right) \leq m,
\end{aligned}
$$

Values of inter-criteria parameters:

$0 \leq \lambda \leq 1$

$\sum_{j=1}^{m} \psi_{j}\left(a_{j}^{*}, a_{j, *}\right)=1$, for all $j=1, \ldots, m:\left(g_{j}\left(a_{j}^{*}\right)=x_{j}^{m_{j}}\right)$ and $\left(g_{j}\left(a_{j, *}\right)=x_{j}^{0}\right)$

with $a_{j}^{*}, a_{j, *} \in A, j=1, \ldots, m$,

$v_{j}(a) \geq p_{j}^{R}(a)+\varepsilon, j=1, \ldots, m, \quad$ for all $a \in A$,

$v_{j}(a) \geq g_{j}(b)-g_{j}(a)+\varepsilon$ if $a \sim_{j} b$, and $g_{j}(a) \leq g_{j}(b), j \in G_{2}$,

$v_{j}(a) \geq v_{j}(b)$ if $g_{j}(a)>g_{j}(b), j=1, \ldots, m, \quad$ for all $(a, b) \in A \times A$,

$v_{j}(a)=v_{j}(b)$ if $g_{j}(a)=g_{j}(b), j=1, \ldots, m, \quad$ for all $(a, b) \in A \times A$,

Values of marginal concordance indices conditioned by intra-criterion preference information:

$$
\begin{aligned}
& \psi_{j}(a, b)=0 \text { if } g_{j}(a)-g_{j}(b) \leq-p_{j}^{R}(a), \text { for all }(a, b) \in A \times A, j \in G_{1}, \\
& \psi_{j}(a, b) \geq \varepsilon \text { if } g_{j}(a)-g_{j}(b)>-p_{j}^{L}(a), \text { for all }(a, b) \in A \times A, j \in G_{1}, \\
& \psi_{j}(a, b)=\psi_{j}\left(a_{j}^{*}, a_{j, *}\right) \text { if } g_{j}(a)-g_{j}(b) \geq-q_{j}^{L}(a), \text { for all }(a, b) \in A \times A, j \in G_{1}, \\
& \psi_{j}(a, b)+\varepsilon \leq \psi_{j}\left(a_{j}^{*}, a_{j, *}\right) \text { if } g_{j}(a)-g_{j}(b)<-q_{j}^{R}(a), \text { for all }(a, b) \in A \times A, j \in G_{1}, \\
& \psi_{j}(a, b)=\psi_{j}\left(a_{j}^{*}, a_{j, *}\right), \psi_{j}(b, a)=\psi_{j}\left(a_{j}^{*}, a_{j, *}\right) \text { if } a \sim_{j} b, j \in G_{2}, \\
& \psi_{j}(a, b)=0 \text { if } b \succ_{j} a, j \in G_{2},
\end{aligned}
$$

Monotonicity of the functions of marginal concordance indices:

If the thresholds for $g_{j}$ are not dependent on $g_{j}(a)$ :

$\psi_{j}(a, b) \geq \psi_{j}(c, d)$ if $g_{j}(a)-g_{j}(b)>g_{j}(c)-g_{j}(d)$, for all $a, b, c, d \in A, j=1, \ldots, m$, $\psi_{j}(a, b)=\psi_{j}(c, d)$ if $g_{j}(a)-g_{j}(b)=g_{j}(c)-g_{j}(d)$, for all $a, b, c, d \in A, j=1, \ldots, m$,

If the thresholds for $g_{j}$ are dependent on $g_{j}(a)$ :

$\psi_{j}(a, c) \geq \psi_{j}(b, c)$ if $g_{j}(a)>g_{j}(b)$, for all $a, b, c \in A, j=1, \ldots, m$,

$\psi_{j}(a, c) \geq \psi_{j}(b, c)$ if $g_{j}(a)=g_{j}(b)$, for all $a, b, c \in A, j=1, \ldots, m$,

$\psi_{j}(a, b) \geq \psi_{j}(a, c)$ if $g_{j}(b)<g_{j}(c)$, for all $a, b, c \in A, j=1, \ldots, m$,

$\psi_{j}(a, b) \geq \psi_{j}(a, c)$ if $g_{j}(b)=g_{i}(c)$, for all $a, b, c \in A, j=1, \ldots, m$. 
Analogously to what has been done in Section 4.1, to verify the feasibility of this set of constraints $E^{A^{R^{\prime}}}$ by linear programming, one has to transform the strict inequality constraints into weak inequalities involving a variable $\varepsilon$. $E^{A^{R^{\prime}}}$ has the form of 0-1 mixed integer program (MIP). If $E^{A^{R^{\prime}}}$ is feasible and $\varepsilon^{*}>0$, where $\varepsilon^{*}=\max \varepsilon$, subject to $E^{A^{R^{\prime}}}$, then there exists at least one instance of the preference model compatible with the preference information provided by the DM.

\section{Appendix B (Computation of the relations $S^{N}$ and $S^{P}$ )}

Given a pair of alternatives $(a, b) \in A \times A$, and the following sets of constraints

$$
\begin{aligned}
& E^{A^{R^{\prime}}} \\
& \left.\begin{array}{l}
C(a, b)=\sum_{i=1}^{n} \psi_{i}(a, b)+\varepsilon \leq \lambda+M_{0}(a, b) \text { and } g_{i}(b)-g_{i}(a) \geq v_{i}(a)-\delta M_{i}(a, b), \\
\sum_{i=0}^{n} M_{i}(a, b) \leq n, M_{i}(a, b) \in\{0,1\}, i=0, \ldots, n
\end{array}\right\} E^{S^{N}}(a, b) \\
& \quad \text { and } \\
& E^{A^{R^{\prime}}} \\
& \left.C(a, b)=\sum_{i=1}^{n} \psi_{i}(a, b) \geq \lambda \text { and } g_{i}(b)-g_{i}(a)+\varepsilon \leq v_{i}(a), i=1, \ldots, n\right\} E^{S^{P}}(a, b)
\end{aligned}
$$$$
\text { we have the following: }
$$

- $a S^{N} b$ iff $E^{S^{N}}(a, b)$ is infeasible or $\varepsilon^{N}(a, b) \leq 0$, where $\varepsilon^{N}(a, b)=\max \varepsilon$ subject to $E^{S^{N}}(a, b)$,

- $a S^{P} b$ iff $E^{S^{P}}(a, b)$ is feasible and $\varepsilon^{P}(a, b)>0$, where $\varepsilon^{P}(a, b)=\max \varepsilon$ subject to $E^{S^{P}}(a, b)$. 